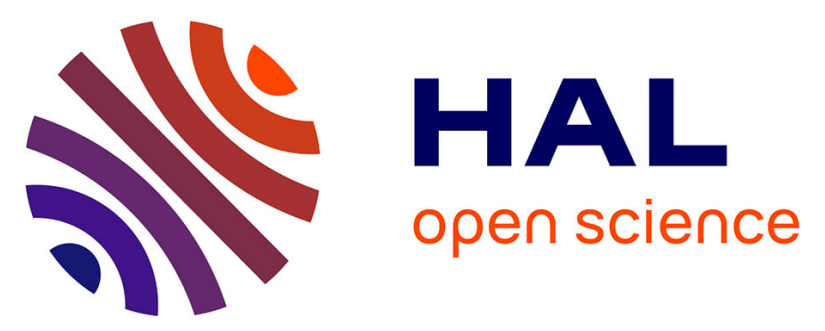

\title{
Electron and Lattice Heating Contributions to the Transient Optical Response of a Single Plasmonic Nano-Object
}

Romain Rouxel, Michele Diego, Paolo Maioli, Noelle Lascoux, Fabien Vialla, Francesco Rossella, Francesco Banfi, Fabrice Vallée, Natalia del Fatti, Aurélien Crut

\section{To cite this version:}

Romain Rouxel, Michele Diego, Paolo Maioli, Noelle Lascoux, Fabien Vialla, et al.. Electron and Lattice Heating Contributions to the Transient Optical Response of a Single Plasmonic Nano-Object. Journal of Physical Chemistry C, 2021, 125 (42), pp.23275-23286. 10.1021/acs.jpcc.1c06629 . hal03410839

\section{HAL Id: hal-03410839 \\ https://hal.science/hal-03410839}

Submitted on 2 Nov 2021

HAL is a multi-disciplinary open access archive for the deposit and dissemination of scientific research documents, whether they are published or not. The documents may come from teaching and research institutions in France or abroad, or from public or private research centers.
L'archive ouverte pluridisciplinaire HAL, est destinée au dépôt et à la diffusion de documents scientifiques de niveau recherche, publiés ou non, émanant des établissements d'enseignement et de recherche français ou étrangers, des laboratoires publics ou privés. 


\title{
Electron and Lattice Heating Contributions to the Transient Optical Response of a Single Plasmonic Nano-Object
}

\author{
Romain Rouxel ${ }^{1}$, Michele Diego ${ }^{1}$, Paolo Maioli ${ }^{1}$, Noelle Lascoux ${ }^{1}$, Fabien Vialla ${ }^{1}$, Francesco Rossella ${ }^{2}$, \\ Francesco Banfi ${ }^{1}$, Fabrice Vallé ${ }^{1}$, Natalia Del Fatti ${ }^{1{ }^{*}}$, and Aurélien Crut ${ }^{1}$ \\ ${ }^{1}$ FemtoNanoOptics group, Université de Lyon, CNRS, Université Claude Bernard Lyon 1, Institut Lumière \\ Matière, F-69622 Villeurbanne, France \\ ${ }^{2}$ NEST, Scuola Normale Superiore and Istituto Nanoscienze-CNR, Piazza S. Silvestro 12, I-56124 Pisa, \\ Italy
}

\begin{abstract}
The sudden absorption of light by a metal nanoparticle launches a series of relaxation processes (internal thermalization, acoustic vibrations and cooling) which induce a transient modification of its optical response. In this work, the transient optical response associated to the internal thermalization of a single gold nanodisk (occurring on a few picoseconds timescale) was quantitatively investigated by time-resolved spectroscopy experiments, and the measured signals were compared with a model accounting for the effects of both electron and ionic lattice heating. We show that experimental timeresolved signals at delays posterior to nanodisk excitation and electron gas thermalization can be simply interpreted as a combination of electron and lattice temperature evolutions, with probe wavelength-dependent weights. This demonstrates the possibility to selectively probe the electronic or lattice dynamics, through the choice of specific probe wavelengths. Additionally, the timedependent spectral shape of transient extinction cross-section changes is shown to be successively dominated by the effect of electron and lattice heating, which present distinct spectral signatures.
\end{abstract}

KEYWORDS: single nano-objects, time-resolved spectroscopy, transient optical response, gold nanoparticles, electron-phonon energy exchanges, nanoplasmonics 


\section{INTRODUCTION}

The impulsive excitation of a metal nano-object induces a series of ultrafast relaxation processes, including internal thermalization by electron-electron and electron-phonon scattering, acoustic vibrations and thermal cooling. All these phenomena have generated a large interest, as they enable to address fundamental nanoscience questions (e.g., regarding the dependence of their associated kinetics on nano-object size) and allow the development of applications based for instance on the use of nano-objects for catalytic purposes or as generators of heat and $\mathrm{GHz} / \mathrm{THz}$ acoustic waves. ${ }^{1-7}$ Optical pump-probe spectroscopy constitutes a powerful approach to experimentally investigate these processes. It is based on the excitation of nano-objects by a femtosecond "pump" pulse and the monitoring of their subsequent relaxation dynamics using a second time-delayed femtosecond "probe" pulse, and enables the investigation of the dynamic processes occurring on femtosecond to nanosecond timescales, provided they have an impact on the optical response of the investigated nano-objects. Many experimental investigations on ensembles of metal nano-objects (in most cases colloidal solutions or glass samples containing gold or silver nanoparticles) have been performed in the last 25 years. They have greatly clarified the kinetics of the phenomena mentioned above, and their dependences on the properties of the nanoparticles (composition, morphology, crystallinity...) and of their local environment. ${ }^{8-16}$

The introduction of single metal nanoparticle optical spectroscopy techniques at the beginning of the $21^{\text {th }}$ century has represented an important breakthrough in this research field. ${ }^{17-22}$ Indeed, singleparticle experiments avoid all the spurious effects associated to dispersions of nanoparticle size, shape and environment affecting measurements on ensembles of nanoparticles. In particular, this approach allows more detailed studies of damping processes, and also enables to precisely quantify the transient changes of the optical cross-sections of a nanoparticle occurring after its sudden excitation, and to correlate them with the nanoparticle's morphology and static optical properties. Up to now, singleparticle time-resolved spectroscopy techniques have been mostly exploited for the investigation of the vibrational damping of nano-objects, allowing significant progress in the understanding of this phenomenon. ${ }^{7,23-28}$ They were also applied - although less frequently - to studies of the internal thermalization ${ }^{21,29,30}$ and cooling dynamics ${ }^{31}$ of metal nanoparticles.

The aim of the work described in this paper was to quantitatively investigate the contributions of electron and lattice heating to the transient optical response of a single plasmonic nanoparticle at short timescales preceding its internal thermalization, i.e., when its state cannot be defined by a single temperature (Figure 1). To do so, time-resolved experiments with pump-probe delays of up to $10 \mathrm{ps}$ were performed on single gold nanodisks (NDs). They were analyzed in the framework of a theoretical 
model addressing the dynamics and influence on the gold dielectric function of both electron and lattice heating, and constituting a refined version of that used in a previous work, performed on single plasmonic nanoparticles with a different shape (single gold nanorods). ${ }^{29}$ Comparison of the measured signals with this more complete model enables to clarify the contributions of electron and lattice heating to the transient optical response of the investigated NDs at all considered timescales. We show that, for delays posterior to the excitation and the thermalization of the electron gas, time-resolved signals generally reflect a combination of electron and lattice temperature evolutions (whose typical temporal profiles are shown in Figure 1b), with probe wavelength-dependent weights. We then demonstrate that these distinct dynamics can both be selectively probed through the use of specific probe wavelengths.

\section{EXPERIMENTAL AND THEORETICAL METHODS}

Nanodisk Fabrication and Morphological Characterization. Gold NDs were nanopatterned by a combination of electron beam lithography, Au thermal evaporation, and lift-off techniques on the optically polished surface of a $480 \mu \mathrm{m}$ thick (0001) $\alpha-\mathrm{Al}_{2} \mathrm{O}_{3}$ single-crystal sapphire substrate. ${ }^{27,31,32}$ Atomic force and scanning electron microscopy characterizations were performed to estimate their thicknesses (about $20 \mathrm{~nm}$ ) and diameters (about 100 to $150 \mathrm{~nm}$ for the NDs considered in this work), respectively.

Spatial Modulation Microscopy/Spectroscopy. Localization and extinction spectroscopy of individual NDs were performed by spatial modulation spectroscopy (SMS), a far-field optical technique based on the periodic displacement of a single nano-object in the focal spot of a tightly focused light beam, which enables the extinction cross-section $\sigma_{\text {ext }}$ of single nano-objects to be quantitatively determined as a function of the illumination wavelength $\lambda$ and light polarization angle $\theta \cdot{ }^{18,33}$ SMS experiments were performed using a tunable Ti:sapphire oscillator combined with a visible optical parametric oscillator and a frequency-doubling BBO crystal as a light source, enabling optical studies in the 375-1040 nm wavelength range. The light beam delivered by this source was focused on the sample by a $100 \mathrm{X}$ microscope objective, with the direction of its linear polarization being controlled by a wire grid polarizer. The size of the laser spot at the sample surface, close to the diffraction limit (about $0.7 \lambda$ fullwidth at half-maximum), was precisely determined by two-dimensional fitting of the SMS images of small NDs obtained using an XY piezoelectric translation stage. Spatial modulation of the sample was performed at $\mathrm{f}=1.5 \mathrm{kHz}$ frequency with $300 \mathrm{~nm}$ modulation amplitude in the $\mathrm{Y}$ direction, and lock-in detection at 2f. Quasicircular NDs, identified from the weak dependence of their extinction on $\theta,{ }^{32}$ were selected for the present study. 
Time-Resolved Experiments. The transient optical response of individual gold NDs was measured using single-particle femtosecond pump-probe spectroscopy, ${ }^{21,29}$ an approach based on the illumination of the investigated nanoparticle with two spatially overlapping, tightly focused, and time-delayed femtosecond laser pulses, referred to as pump and probe. Time-resolved measurements on individual NDs were performed by combining the SMS microscope with a two-color pump-probe setup based on the femtosecond laser source described in the previous paragraph. The oscillator pulse train was split in two parts to generate the pump and probe beams. The pump beam was produced using second harmonic generation (yielding $\lambda_{\mathrm{pp}}$ in the $375-510 \mathrm{~nm}$ range), whereas the probe beam was either the signal from the Ti:sapphire oscillator (with $\lambda_{\mathrm{pr}}=750-1020 \mathrm{~nm}$ in this case) or the output of the optical parametric oscillator $\left(\lambda_{\mathrm{pr}}=500-730 \mathrm{~nm}\right.$, with here $\left.\lambda_{\mathrm{pp}}=410 \mathrm{~nm}\right)$. Durations of approximately $1 \mathrm{ps}$ and $200 \mathrm{fs}$ were measured for the pump and probe pulses, respectively (the relatively long duration of pump pulses in the blue spectral region originating from their broadening through the microscope objective used in this work). A mechanical chopper operating at $30 \mathrm{kHz}$ was used for pump power modulation. The pump-induced relative changes of the probe beam transmission, $\Delta T r / T r$, were measured using synchronous detection as a function of the time interval separating the pump and probe pulses, controlled by a mechanical delay line. This raw measured signal is directly related to the transient changes of the extinction cross-section $\sigma_{\text {ext }}\left(\lambda_{\text {pr }}\right)$ of the investigated nano-object at probe wavelength $\lambda_{\text {pr }}$ via $\Delta \operatorname{Tr} / \operatorname{Tr}=-\Delta \sigma_{\text {ext }}\left(\lambda_{\text {pr }}\right) / S_{p r}$, with $S_{p r}=\pi d_{f w h m}{ }^{2} /(4 \ln 2)$ representing the area of the probe spot and $d_{\text {fwhm }}$ its full-width at half-maximum..$^{29}$ As $S_{p r}$ can be estimated by analysis of SMS images, experiments provide access to the ultrafast variations of $\Delta \sigma_{\text {ext }}$ as a function of the pump-probe delay. The temporal origin of the signals, defined as the instant at which the pump and probe pulses present a maximal overlap, was a posteriori determined for each signal by comparison with the complete model presented below.

Modeling of the gold dielectric function. The dielectric function $\varepsilon=\varepsilon_{1}+i \varepsilon_{2}$ of bulk gold in the visible/near-infrared range can be written as the sum of two frequency-dependent components, related to interband (ib) and intraband (Drude-like) absorption mechanisms ${ }^{33-35}$ :

$$
\varepsilon(\omega)=\varepsilon^{i b}(\omega)+\varepsilon^{D}(\omega)-1=\varepsilon^{i b}(\omega)-\frac{\omega_{p}^{2}}{\omega(\omega+i \gamma)}
$$

with $\omega_{p}$ the plasma frequency ( $\hbar \omega_{p}=9.01 \mathrm{eV}$ for bulk gold) and $\gamma$ the optical scattering rate of the conduction electrons ( $\hbar \gamma \approx 50 \mathrm{meV}$ for bulk gold). In this work, $\varepsilon$ was modeled based on Johnson and Christy tables of the bulk gold dielectric function $\varepsilon_{\mathrm{JC} .}{ }^{36}$ The interband component of the bulk gold dielectric function at room temperature, deduced from $\varepsilon_{\mathrm{Jc}}$ tables as $\varepsilon^{i b}=\varepsilon_{J C}+\frac{\omega_{p}{ }^{2}}{\omega(\omega+i \gamma)}$, was theoretically reproduced using the approach developed by Rosei ${ }^{37}$ and the gold band structure 
calculated by Rangel et al. ${ }^{38}$ The imaginary part of $\varepsilon^{i b}, \varepsilon_{2}^{i b}(\omega)$ was obtained by evaluating the total probability of a transition from an occupied state to an empty one by absorption of a photon of energy $\hbar \omega$, considering electronic transitions around the $L$ and $X$ points of the first Brillouin zone. The procedure described above, initially used to compute the interband component of the gold dielectric function for a Fermi-Dirac electron distribution at room temperature, can be easily reiterated for different electronic temperatures and non-thermal (i.e., non Fermi-Dirac) electronic distributions, thus allowing to predict the temporal evolution of $\varepsilon^{i b}$ in the context of time-resolved experiments, by computing $\Delta \varepsilon_{2}^{i b}(t)$ and deducing $\Delta \varepsilon_{1}^{i b}(t)$ through the Kramers-Kronig relations (see below). ${ }^{40}$

Modeling of the linear optical response of gold NDs. Modeling of the ND linear optical properties was performed by finite-element modeling (FEM), using the RF module of the COMSOL commercial software. Gold NDs were described as circular cylinders characterized by their thickness $h$ and diameter $D$. The sapphire substrate supporting them was explicitly included in the model. Refractive indexes of 1.76 and 1 were used for the sapphire substrate and the medium above it, respectively. A perfectly matched layer was used around the simulation domain to avoid spurious finite-size effects. The dielectric function of gold was described using eq 1 , using for $\omega_{p}$ and $\varepsilon^{i b}$ their bulk values but leaving $\gamma$ as a free parameter (using $\hbar \gamma$ values larger than the bulk gold value is often necessary to reproduce the SPR width measured on single nano-objects, and physically corresponds to taking into account additional non-radiative plasmon damping mechanisms in metal nano-objects as compared to the bulk metal case ${ }^{41-44}$ ). ND illumination by a linearly polarized plane wave was considered. Absorption and scattering cross-sections were deduced from the computed electric field, and summed to yield the extinction one $\sigma_{\text {ext. }}$. The $D$ and $h$ values used in the modeling were adjusted so as to optimize the agreement with the investigated experimental spectrum (Figure 2a). The $\partial \sigma_{\text {ext }} / \partial \varepsilon_{1}$ and $\partial \sigma_{\text {ext }} / \partial \varepsilon_{2}$ partial derivatives (Figure $2 \mathrm{~b}$ ) were deduced from these calculations by computing $\sigma_{\text {ext }}$ with slightly modified $\varepsilon_{1}$ or $\varepsilon_{2}$ values and then performing discrete derivation.

Modeling of the transient optical response of gold NDs. Complete modeling of the transient optical response of gold NDs following their impulsive excitation was performed along the following steps. - Evolution of the energy- and time-dependent distribution function $f(E, t)$ (where $E$ refers to the energy of the electronic state) of gold quasi-free electrons in the conduction band was computed in the framework of a bulk-like approach by numerically solving Boltzmann equation, ${ }^{39,40}$ including the effects of three terms, corresponding to initial pulse excitation (leading to an initially athermal electron distribution), thermalization of the electron gas by electron-electron scattering (occurring on a timescale of a few hundred fs) and electron-to-lattice energy transfer by electron-phonon scattering (complete after a few ps).

- The electron excess energy density $\Delta \mathrm{u}_{\mathrm{e}}(\mathrm{t})$ and equivalent temperature increase $\Delta \mathrm{T}_{\mathrm{e}}(\mathrm{t})$ are deduced 
from $\Delta f(E, t)=f(E, t)-f_{0}(E)$, with $f_{0}$ the initial electronic Fermi-Dirac distribution at room temperature $\left(T_{0}=295 \mathrm{~K}\right)$. The lattice excess energy density $\Delta \mathrm{u}_{\mathrm{L}}(\mathrm{t})$ and temperature increase $\Delta \mathrm{T}_{\mathrm{L}}(\mathrm{t})$ are also computed at each time-step by applying energy conservation for the \{electron+lattice system (see below for more details).

- Knowledge of $\Delta f(E, t)$ and $\Delta T_{L}(t)$ enables the transient change $\Delta \varepsilon(t)$ of the ND dielectric function to be determined at each time-step, by separately computing and summing its interband and Drude-like components.

- Combining $\Delta \varepsilon(\mathrm{t})$ with the $\partial \sigma_{\mathrm{ext}} / \partial \varepsilon_{1}$ and $\partial \sigma_{\mathrm{ext}} / \partial \varepsilon_{2}$ derivatives deduced from FEM simulations (see section Modeling of the linear optical response of gold NDs) allows to quantitatively predict the transient variations $\Delta \sigma_{\text {ext }}\left(\lambda_{p r}, t\right)$ of the gold ND extinction cross-section, the quantity measured in the context of time-resolved experiments.

The average measured values of pump and probe pulse durations (about 1 ps and $200 \mathrm{fs}$ ) were considered in the modeling, for the time-dependent excitation of the electron gas and for convoluting the computed $\Delta \sigma_{\text {ext }}(\mathrm{t})$, respectively. A 500 fs time constant was assumed for the electron gas thermalization by electron-electron interactions in gold, ${ }^{40}$ while the electron-to-lattice energy transfer time $\left(\approx 1\right.$ ps in gold in the weak-excitation regime $\left.{ }^{9,45,46}\right)$ was left as a free parameter, as its value for nanoparticles produced by electron beam lithography may be affected by the presence of crystalline defects. ${ }^{47,48}$ The ND thermal cooling induced by heat propagation in the substrate, investigated in detail in a recent study, ${ }^{31}$ was neglected here as it occurs on timescales of about 500 ps for $20 \mathrm{~nm}$ thick NDs, much longer than the $<10$ ps ones investigated here.

Electronic and lattice energy densities and temperatures. In more details, the excitation process leads

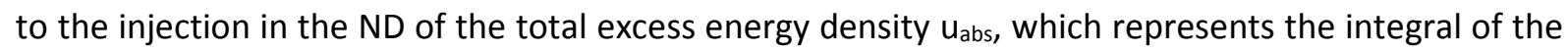
instantaneous absorbed power density $p_{a b s}$ over the pump pulse duration. $u_{a b s}$ is computed as $u_{a b s}=$ $\sigma_{a b s} F_{p p} / V$, with $\sigma_{a b s}$ and $V$ being the ND absorption cross-section and volume, deduced from FEM optical simulations, and $F_{p p}=P_{p p} /\left(f_{r e p} S_{p p}\right)$ the fluence of the pump beam $\left(60 \mu \mathrm{J} / \mathrm{cm}^{2}\right.$ average value in our experiments), with $P_{p p}$ and $S_{p p}$ its average power and focal spot size, and $f_{r e p}$ the repetition rate of the laser source. An excitation temperature $T_{\text {exc }}$ (the temperature that the electron gas would achieve at the end of its thermalization, in the absence of electron-phonon coupling) can be defined through the relation $u_{a b s}=\int_{T_{0}}^{T_{e x c}} c_{e}\left(T_{e}\right) d T_{e}$, yielding $T_{e x c}=\sqrt{T_{0}{ }^{2}+\frac{2 u_{a b s}}{a}}$, with $\mathrm{c}_{\mathrm{e}}=\mathrm{aT}$ e the volumetric electronic heat capacity ( $\mathrm{a} \approx 65 \mathrm{~J} \cdot \mathrm{K}^{-2} \cdot \mathrm{m}^{-3}$ for gold). Similarly, an equivalent temperature (i.e., associated to a Fermi-Dirac distribution with same excess energy) can be defined from $\Delta \mathrm{u}_{\mathrm{e}}(\mathrm{t})$, the time-dependent excess energy density in the electron gas, as $T_{e}(t)=\sqrt{T_{0}{ }^{2}+\frac{2 \Delta u_{e}(t)}{a}}$. The increase of the lattice temperature (assumed to remain well-defined throughout the experiments), $\Delta T_{L}(t)$, is calculated by 
considering that the energy lost by the electrons is entirely transferred to the lattice (the temperaturedependence of the lattice volumetric heat capacity $C_{L}$ being neglected, due to the small $\Delta T_{L} \approx 10 \mathrm{~K}$ maximal lattice heating in our experiments). We note $\Delta T_{\text {eq }}$ the equilibrium temperature rise of the ND after its full internal thermalization. ${ }^{49}$ An example of the temporal evolutions of $p_{a b s}, \Delta u_{e}, \Delta u_{L}, \Delta T_{e}$ and $\Delta T_{L}$ computed using pump pulse and nano-object parameters relevant for the experiments described in this paper can be found in Figure $1 \mathrm{~b}$.

Transient variations of the gold dielectric function. $\varepsilon$ (eq 1; note that the spectral dependence of $\varepsilon(\omega)$ is not reminded in this paragraph for the sake of simplicity) is a function of both the electronic distribution $f(E)$ and the lattice temperature $T_{L}$. As $\Delta T_{L}$ values are small in our experiments (typically less than $10 \mathrm{~K}$ ), the dependence of $\varepsilon$ on $T_{L}=T_{0}+\Delta T_{L}$ can be linearized as $\varepsilon\left(f, T_{L}\right) \approx \varepsilon\left(f, T_{0}\right)+\Delta T_{L} \frac{\partial \varepsilon}{\partial T_{L}}\left(f, T_{0}\right)$. Moreover, numerical investigations based on the model described in ref. ${ }^{39}$ showed that the $\frac{\partial \varepsilon}{\partial T_{L}}\left(\mathrm{f}, \mathrm{T}_{0}\right)$ derivative does not strongly depend on the electronic distribution, and can thus be approximated by $\frac{\partial \varepsilon}{\partial T_{L}}\left(\mathrm{f}_{0}, \mathrm{~T}_{0}\right)$. Under this approximation, the modifications of $\varepsilon$ induced by electron and lattice heating contributions can be fully separated. The transient change $\Delta \varepsilon(t)$ of the gold dielectric function can be decomposed as the sum of an electronic contribution, $\Delta \varepsilon_{\mathrm{e}}(\mathrm{t})$, resulting from the modification of the electronic distribution $f(t)$, and of a lattice one, $\Delta \varepsilon_{L}(t)$, resulting from the increase of the lattice temperature $\Delta T_{L}(t)$ :

$$
\Delta \varepsilon(t)=\Delta \varepsilon_{e}(t)+\Delta \varepsilon_{L}(t)
$$

with

$$
\left\{\begin{array}{c}
\Delta \varepsilon_{e}(t)=\varepsilon\left(f(t), T_{0}\right)-\varepsilon\left(f_{0}, T_{0}\right)=\Delta \varepsilon_{e}^{i b}(t)+\Delta \varepsilon_{e}^{D}(t) \\
\Delta \varepsilon_{L}(t)=\frac{\partial \varepsilon}{\partial T_{L}}\left(f_{0}, T_{0}\right) \cdot \Delta T_{L}(t)=\Delta \varepsilon_{L}^{i b}(t)+\Delta \varepsilon_{L}^{D}(t)
\end{array}\right.
$$

, the equalities on the right of eq 3 corresponding to the decomposition of $\Delta \varepsilon_{e, L}$ into interband and Drude components (eq 1). Note that, in the formulation of eq. $3, \Delta \varepsilon_{L}(t)$ is proportional to $\Delta T_{L}(t)$ while the dependence of $\Delta \varepsilon_{\mathrm{e}}(\mathrm{t})$ on the electronic temperature is more complex.

Electronic contribution to $\Delta \varepsilon\left(\Delta \varepsilon_{e}\right)$. The $\Delta \varepsilon_{e}^{i b}(\mathrm{t})$ term appearing in eq 3 , associated to the modification of the probabilities of interband transitions to and from the conduction band caused by the evolution of the electron distribution $f(E, t)$, was computed by reproducing the calculations discussed in the paragraph Modeling of the gold dielectric function with the $f(E, t)$ distribution functions obtained at multiple instants $\mathrm{t}$ by solving the Boltzmann equation. Modification of the electronic distribution additionally generates a $\Delta \varepsilon_{e}^{D}(\mathrm{t})$ change of the Drude component of $\varepsilon$ (eq 3 ), dominated by the modification of the electron-electron scattering rate $\gamma_{\mathrm{e}-\mathrm{e}}$, which contributes to the overall scattering 
rate $\gamma$ appearing in the Drude component of $\varepsilon$ and mainly affecting its imaginary part (eq 1$)$. The variation of the electron-electron scattering rate has been predicted to be quadratic with the electron temperature $\mathrm{T}_{\mathrm{e}}: 39,50$

$$
\frac{\Delta \gamma_{e-e}}{\gamma_{e-e}}=\left(\frac{2 \pi k_{B}}{\hbar \omega}\right)^{2}\left(T_{e}{ }^{2}-T_{0}^{2}\right)
$$

with $\mathrm{Y}_{\mathrm{e}-\mathrm{e}} \approx 15 \mathrm{meV}$ at room temperature in the visible range $\mathrm{e}^{51}$-a value however affected by a large uncertainty, as measurement of the individual contribution of electron-electron scattering to the overall scattering rate $y$ is challenging. In this work, eq 4 was used both in the thermal and athermal regimes, using the equivalent electronic temperature in the latter case.

Lattice contribution to $\Delta \varepsilon\left(\Delta \varepsilon_{L}\right)$. Lattice heating induces a modification of both $\varepsilon^{\text {ib }}$ (by lattice dilation, $\Delta \varepsilon_{L}^{i b}(\mathrm{t})$ term in eq 3 ) and $\varepsilon^{\mathrm{D}}$ (by modification of the plasma frequency $\omega_{p}$ and scattering rate $\gamma$ appearing in eq 1 , respectively caused by the dilation-induced change of electron density and by the $\mathrm{T}_{\mathrm{L}}$-dependence of electron-phonon scattering rate, $\Delta \varepsilon_{L}^{D}(\mathrm{t})$ term in eq 3). As in the small $\Delta \mathrm{T}_{\mathrm{L}}$ regime considered here $\Delta \varepsilon_{L}$ can be assumed to depend linearly on $\Delta T_{L}$ (eqs 2 and 3 ), the optical consequences of lattice heating can therefore be summarized by a single $d \varepsilon / d T_{L}$ spectrum. In this work, we used the $\mathrm{d} \varepsilon_{1,2} / \mathrm{d} \mathrm{T}_{\mathrm{L}}$ tables derived by Stoll et al. from a theoretical model accounting for the effects mentioned above, ${ }^{39}$ which were shown to allow a good reproduction of the thermo-optical dynamics of single gold NDs in the regime of large time delays, for which their electronic and lattice temperatures coincide. ${ }^{31}$ $\Delta \varepsilon$-induced transient changes of ND extinction cross-section. $\Delta \sigma_{\mathrm{ext}}\left(\lambda_{\mathrm{pr}}, \mathrm{t}\right)$ was deduced from the computed gold dielectric function changes $\Delta \varepsilon_{1,2}\left(\lambda_{p r}, t\right)$ and $\varepsilon_{1,2}$ derivatives of ND extinction crosssection as:

$$
\Delta \sigma_{e x t}\left(\lambda_{p r}, t\right)=\frac{\partial \sigma_{e x t}}{\partial \varepsilon_{1}}\left(\lambda_{p r}\right) \Delta \varepsilon_{1}\left(\lambda_{p r}, t\right)+\frac{\partial \sigma_{e x t}}{\partial \varepsilon_{2}}\left(\lambda_{p r}\right) \Delta \varepsilon_{2}\left(\lambda_{p r}, t\right)
$$

Calculation of $\frac{\partial \sigma_{e x t}}{\partial \varepsilon_{1}}$ and $\frac{\partial \sigma_{e x t}}{\partial \varepsilon_{2}}$ was discussed in the section Modeling of the linear optical response of gold NDs, their values being shown in Figure $2 \mathrm{~b}$ for the ND considered throughout the paper. Eq 5 implies that, similarly to $\Delta \varepsilon$ (eqs 2-3), $\Delta \sigma_{\text {ext }}\left(\lambda_{\text {pr }}, \mathrm{t}\right.$ ) can be decomposed as a sum of two terms (each one being the sum of interband and Drude components, as in eq 3 ) associated to electronic and lattice heating, respectively:

$$
\Delta \sigma_{\text {ext }}\left(\lambda_{p r}, t\right)=\Delta \sigma_{\text {ext,e }}\left(\lambda_{p r}, t\right)+\Delta \sigma_{\text {ext }, L}\left(\lambda_{p r}, t\right)
$$

Simulated time-resolved signals were finally deduced from computed transient extinction changes by performing their time-domain convolution with the intensity profile of the probe pulse, assumed Gaussian. 


\section{RESULTS AND DISCUSSION}

Single gold NDs were investigated by SMS (yielding their absolute extinction cross-section spectrum $\left.\sigma_{\text {ext }}(\lambda)\right)$ and time-resolved spectroscopy with different probe wavelengths $\lambda_{\text {pr }}$ (yielding time- and probe wavelength-dependent transient extinction cross-section variations $\left.\Delta \sigma_{\text {ext }}\left(t, \lambda_{\text {pr }}\right)\right)$. These measurements and their modeling are presented in Figures 2-6 for an illustrative ND (a similar set of measurements on a second ND being presented in Figures S1 to S5 of the Supporting Information). The ND extinction spectrum is shown in Figure 2a. It presents a resonance at $860 \mathrm{~nm}$ central wavelength, corresponding to the ND in plane dipolar localized surface plasmon resonance (SPR), which is well reproduced by optical FEM simulations performed with $D=153 \mathrm{~nm}$ and $h=22 \mathrm{~nm}$, close to the sizes targeted during the fabrication process and those estimated by SEM and AFM imaging. ${ }^{31}$

A selection of time-resolved signals measured on this ND is shown in Figure 3a. As these signals were acquired with different pump beam parameters, leading to slightly different equilibrium temperature rises, they were divided by $\Delta \mathrm{T}_{\text {eq, }}$, which is of the order of $10 \mathrm{~K}$ in our experiments. It should however be noted that this procedure does not fully suppress the $\Delta T_{\text {eq }}$ dependence of time-resolved signals due to the fluence-dependence of the electron-lattice energy transfer time out of the low-perturbation regime ${ }^{46,52,53}$ (as shown in Figure S6 of the Supporting Information), so that comparison of normalized signals is fully relevant only for low or weakly dispersed $\Delta T_{\text {eq }}$ values (as was the case here).

Large variations of amplitudes and signs of the transient extinction changes are observed as a function of the probe wavelength $\lambda_{\text {pr }}$. These signals also display very different dynamics depending on $\lambda_{\text {pr }}$, as more clearly evidenced by Figure $3 b$, where they have been normalized by their maximal absolute amplitudes. The majority of normalized signals show a fast rise at short pump-probe delays, followed by a decay leading to an approximately constant background in the $t=5-8 \mathrm{ps}$ range. However, the values of the normalized signals at $t=8$ ps undergo large variations with $\lambda_{\text {pr }}$, being in some cases close to 1 (e.g., for $\lambda_{\mathrm{pr}}=855 \mathrm{~nm}$ ), close to 0 (for $\lambda_{\mathrm{pr}}=700 \mathrm{~nm}$ ) or slightly negative (for $\lambda_{\mathrm{pr}}=905 \mathrm{~nm}$ ). Additionally, the signal measured with $\lambda_{\mathrm{pr}}=890 \mathrm{~nm}$ presents a very different shape, featuring a slow rise between 0 and 5 ps, followed by a plateau.

Time-resolved signals are plotted in Figure $3 \mathrm{c}$ as a function of $\lambda_{\text {pr }}$ for fixed delays, corresponding to 0.7 , 2.5 and 8 ps. These transient spectra present common general features, $\Delta \sigma_{\text {ext }}$ being positive at short and large wavelengths (below $\approx 700 \mathrm{~nm}$ and above $\approx 900 \mathrm{~nm}$ ), and negative in-between. However, their amplitudes and spectral shapes evolve in time (as more easily observable on the normalized spectra shown in Figure $3 d$ ), with in particular a red-shift at longer delays of the $\Delta \sigma_{\text {ext }}$ minimum around $\lambda_{\text {pr }} \approx 850$ $\mathrm{nm}$ and of the zero crossing at $\lambda_{\mathrm{pr}} \approx 700 \mathrm{~nm}$ and $\approx 900 \mathrm{~nm}$. The shape of transient extinction spectra is close to those of the $\partial \sigma_{\text {ext }} / \partial \varepsilon_{1}$ and $\partial \sigma_{\text {ext }} / \partial \varepsilon_{2}$ derivatives (Figure $2 b$ ) at short and large delays, 
respectively.

The measured transient extinction changes $\Delta \sigma_{\text {ext }}\left(t, \lambda_{\text {pr }}\right)$ were quantitatively compared to the results of the numerical model described in the Methods part, which allows to compute the temporal evolutions of the electronic distribution $f(E, t)$ and of the lattice temperature $T_{L}(t)$ occurring during the ND internal thermalization, as well as the induced transient changes of the gold dielectric function $\Delta \varepsilon=\Delta \varepsilon_{1}+i \Delta \varepsilon_{2}$ and of the ND extinction cross-section $\Delta \sigma_{\text {ext. }}$. This model constitutes a refined version of that used in a previous work which addressed the transient optical response of single gold nanorods, in which only the temporal variations of the $\Delta \varepsilon_{e}^{i b}$ term were quantitatively computed. ${ }^{29}$ Here, two additional ingredients were taken into account to estimate the time-dependent $\Delta \varepsilon$ changes, namely the modification of $\varepsilon$ by lattice heating ( $\Delta \varepsilon_{L}^{i b}$ and $\Delta \varepsilon_{L}^{D}$ terms) and that of the Drude component of $\varepsilon$ by electron heating $\left(\Delta \varepsilon_{e}^{D}\right.$ term) (eqs 2-3).

Measured signals were quantitatively compared with complete calculations based on eqs 2 and 3 performed with $\Delta \mathrm{T}_{\mathrm{eq}}=6.5 \mathrm{~K}$, close to the experimental values for the investigated ND (Figure 4). The global agreement between experimental and simulated signals was found to be optimal for an electron-lattice energy transfer time of $\tau_{\mathrm{e}-\mathrm{L}} \approx 800 \mathrm{fs}$ at weak excitation, a value slightly smaller than the one measured on large diameter colloidal gold nanoparticles ${ }^{9}$, possibly as a result of polycrystallinity effects $^{48}$ (the increase of this time constant for strong excitation, ${ }^{49,53}$ illustrated in Figure 56 of the Supporting Information, is predicted by the numerical model). This initial analysis also showed that the agreement between measured and simulated time-resolved signals (in particular in terms of amplitudes) could be significantly improved in some cases by comparing experimental measurements with time-resolved signals simulated using a slightly different (within $<15 \mathrm{~nm}$ ) $\lambda_{\mathrm{pr}}$ value. Such a fine tuning of $\lambda_{\mathrm{pr}}$ in the modeling is justified by the occasional observation of small evolutions of the linear extinction spectra of the investigated NDs during the course of time-resolved measurements.

The overall agreement between experimental (blue dots in Figure 4) and calculated (green lines) timeresolved signals is good, regarding both their absolute amplitudes and their kinetics, for all probe wavelengths investigated. Moreover, the possibility to distinguish in the computations the $\Delta \sigma_{\text {ext }}$ components associated to electron and lattice heating $\left(\Delta \sigma_{\text {ext,e }}\right.$ and $\Delta \sigma_{\text {ext,L, }}$ eq 6 , shown as dotted red and dashed black lines in Figure 4) permits to clarify the physical origins of the measured transient signals and to explain the particular temporal profiles of some of the signals shown in Figure 4, namely: - Signals decreasing to nearly zero at the end of the ND internal thermalization (such as those shown in Figure $4 a$ and $f$, corresponding to measurements with $\lambda_{p r}=725 \mathrm{~nm}$ and $\lambda_{\mathrm{pr}}=915 \mathrm{~nm}$ ). They correspond to the exceptional case of a very small lattice heating contribution to $\Delta \sigma_{\text {ext. }}$. Such signals thus reflect the successive heating and cooling of the electrons in gold.

- The signal measured at $\lambda_{\mathrm{pr}}=890 \mathrm{~nm}$ (Figure $4 \mathrm{~d}$ ), which presents a slow increase (in absolute value), 
in strong contrast with the rapid rise followed by a decay seen for other experimental signals. It corresponds to the opposite exceptional case of a negligible impact of electron heating on the transient extinction signal. This signal shape, here measured on the investigated gold ND only for a specific $\lambda_{\mathrm{pr}}$ value, is reminiscent of those observed in the context of measurements on aluminum NDs, for which lattice heating was predicted to have a dominant effect in the whole $500-900 \mathrm{~nm} \lambda_{\mathrm{pr}}$ range. ${ }^{47}$

- A third noticeable particular case is that of signals presenting a constant $\Delta \sigma_{\text {ext }}$ background value immediately after the end of the ND excitation and electron thermalization (i.e., after about 2 ps) and prior to full ND internal thermalization, which requires the completion of electron-lattice energy transfer. This case occurs when the temporal variations of $\Delta \sigma_{\text {ext,e }}$ and $\Delta \sigma_{\text {ext,L }}$ induced by electronphonon energy exchanges compensate each other, so that their sum $\Delta \sigma_{\text {ext }}$ remains constant. A timeresolved signal close to this situation was measured with $\lambda_{\mathrm{pr}}=855 \mathrm{~nm}$ (Figure 4c). The physical origin of such signal shape will be further clarified by considering the simplified model introduced below. Other signals presenting less specific shapes are shown in Figure $4 \mathrm{~b}$ and $4 \mathrm{e}$. They are representative of the majority of the time-resolved measurements performed on NDs, for which inclusion of both electron and lattice time-dependent contributions is required to account for the observed kinetics. While previous analyses of short-timescale signals focused on the interband electronic contributions $\left(\Delta \varepsilon_{e}^{i b}\right.$ term in eq 3), leading to the quasi-vanishing of calculated signals at the end of internal thermalization, ${ }^{29}$ the quantitative inclusion of the lattice contribution to time-resolved signals proves crucial in the present case. The interband electronic (Drude) term ( $\Delta \varepsilon_{e}^{D}$ term in eq 3) also added in this work (as compared to ref..$^{29}$ ) has a minor effect for $\lambda_{\text {pr }}$ in the visible range, but improves the agreement between experimental and simulated signals for $\lambda_{\text {pr }}$ in the infrared range.

For a full understanding of time-dependent transient extinction changes, it is useful to relate them to the $\Delta \mathrm{T}_{\mathrm{e}}(\mathrm{t})$ and $\Delta \mathrm{T}_{\mathrm{L}}(\mathrm{t})$ temperature rises respectively quantifying electron and lattice heating. Whereas the lattice heating contribution to $\Delta \varepsilon$ is simply approximated as $\Delta \varepsilon_{\llcorner} \propto \Delta \mathrm{T}_{\mathrm{L}}$ in the model used (eq 3 ), which is justified by the fact that $\Delta \mathrm{T}_{\mathrm{L}}$ remains small in the context of our experiments (Figure 1), the electronic term $\Delta \varepsilon_{\mathrm{e}}$ is more complex as the electronic temperature is not always defined ( $\mathrm{T}_{\mathrm{e}}$ only representing an equivalent temperature in the athermal regime occurring during and shortly after excitation, in which the electron gas has to be described by its full electronic distribution) and increases much more than $T_{L}$, the electronic heat capacity being much smaller than the lattice one (Figure 1 ). The electronic temperature becomes well-defined only at time delays exceeding the time needed for the restoration of a Fermi-Dirac electronic distribution after excitation, i.e. a few times the characteristic duration of electron gas thermalization by electron-electron energy exchanges (about $500 \mathrm{fs}$ in gold $\left.^{40}\right)$. In the resulting two-temperature regime, the state of the gold ND is fully characterized by the two temperatures $T_{e}$ and $T_{L}$, so that a quantitative modeling of the transient optical response 
of the metal only requires determination of the temporal evolutions of $T_{e}(t)$ and $T_{L}(t)$ (which can also be obtained in the framework of the well-known two-temperature model ${ }^{54}$ ) and of the dependence of the gold dielectric function on these two temperatures $\varepsilon\left(T_{e}, T_{L}\right)$.

In the context of our experiments, $\Delta \mathrm{T}_{\mathrm{e}}$ is typically of the order of $100 \mathrm{~K}$ at the beginning of the twotemperature regime (Figure 1). For such electron heating, the non-linear dependence of $\Delta \varepsilon_{\mathrm{e}}$ on $\Delta \mathrm{T}_{\mathrm{e}}$ remains moderate (as shown in Figure S7 of the Supporting Information). It is therefore helpful for a

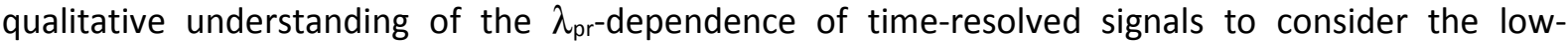
perturbation limit of the complete model used above in the two-temperature regime. In this case, $\Delta \varepsilon_{\mathrm{e}}$ can be written as $\Delta \varepsilon_{e}=\frac{\partial \varepsilon}{\partial T_{e}} \Delta T_{e}$ (such a proportionality between $\Delta \varepsilon_{e}$ and $\Delta T_{e}$ being not assumed to establish eq. 3 , only using $\left.\Delta \varepsilon_{L} \propto \Delta T_{L}\right)$ and eq 5 can then be rewritten as

$$
\Delta \sigma_{e x t}\left(\lambda_{p r}, t\right)=A_{e}\left(\lambda_{p r}\right) \Delta T_{e}(t)+A_{L}\left(\lambda_{p r}\right) \Delta T_{L}(t)
$$

with

$$
\left\{\begin{array}{l}
A_{e}=\frac{\partial \sigma_{\text {ext }}}{\partial T_{e}}=\frac{\partial \sigma_{e x t}}{\partial \varepsilon_{1}} \frac{\partial \varepsilon_{1}}{\partial T_{e}}+\frac{\partial \sigma_{e x t}}{\partial \varepsilon_{2}} \frac{\partial \varepsilon_{2}}{\partial T_{e}} \\
A_{L}=\frac{\partial \sigma_{e x t}}{\partial T_{L}}=\frac{\partial \sigma_{e x t}}{\partial \varepsilon_{1}} \frac{\partial \varepsilon_{1}}{\partial T_{L}}+\frac{\partial \sigma_{e x t}}{\partial \varepsilon_{2}} \frac{\partial \varepsilon_{2}}{\partial T_{L}}
\end{array}\right.
$$

The $\partial \varepsilon_{1,2} / \partial \mathrm{T}_{\mathrm{e}}$ and $\partial \varepsilon_{1,2} / \partial \mathrm{T}_{\mathrm{L}}$ spectra of gold (obtained by calculation of $\Delta \varepsilon_{\mathrm{e}}$ and $\Delta \varepsilon_{\mathrm{L}}$ spectra for small $\Delta \mathrm{T}_{\mathrm{e}}$ and $\Delta T_{L}$ values using our model, followed by numerical derivation), and the $A_{e}$ and $A_{L}$ coefficients obtained for the illustrative ND (using the $\partial \sigma_{\text {ext }} / \partial \varepsilon_{1,2}$ derivatives shown in Figure $2 b$ ) are shown in Figure 5. Note that the $A_{e}$ and $A_{L}$ coefficients of a nanoparticle introduced by eqs 7 and 8 depend on its intrinsic properties (morphology and composition), but are independent of excitation features (pump beam wavelength and fluence). While $\left|A_{L}\right|$ is maximal in the SPR range (where the $\partial \sigma_{\text {ext }} / \partial \varepsilon_{1,2}$ derivatives are the largest, Figure $2 \mathrm{~b}$ ), $\left|\mathrm{A}_{\mathrm{e}}\right|$ is maximal near $500 \mathrm{~nm}$ (where the $\mathrm{T}_{\mathrm{e}}$-derivatives of $\varepsilon_{1}$ and $\varepsilon_{2}$ present large values, Figure 5a), and presents different spectral features associated to the gold interband transitions, in addition to the one in the SPR range. $\left|A_{e}\right|$ presents much smaller values than $\left|A_{L}\right|$ for most wavelengths, except close to the interband spectral region, which implies that the transient extinction changes $\Delta \sigma_{\text {ext }}=\left(A_{e}+A_{L}\right) \Delta T \approx A_{L} \Delta T$ of an internally thermalized $N D\left(\Delta T_{e}=\Delta T_{L}=\Delta T\right)$ are largely dominated by the $\Delta \sigma_{\mathrm{ext}, \mathrm{L}}$ component.

Eq 7 allows a simple qualitative interpretation of measured time-resolved signals in the twotemperature regime, clarifying the origin of their highly variable amplitudes and shapes (Figure $3 a-b$ and Figure 4). Indeed, according to eq 7, time-domain signals represent a linear combination of the electron and lattice temperature evolutions (Figure 1) with probe wavelength-dependent weights, 
both vanishing for specific $\lambda_{\text {pr }}$ values (Figure 4). The particular signals of Figure 4a and $f$ (corresponding to $\lambda_{\mathrm{pr}}=725 \mathrm{~nm}$ and $\lambda_{\mathrm{pr}}=915 \mathrm{~nm}$ ) discussed above, reflecting electronic heating and cooling, can be simply interpreted as resulting from the use of $\lambda_{\text {pr }}$ values close to those for which the AL coefficient is predicted to vanish $\left(\lambda_{\mathrm{pr}} \approx 710\right.$ and $930 \mathrm{~nm}$, Figure $\left.5 \mathrm{~d}\right)$, in the narrow spectral range where $\left|A_{e}\right|$ is not much smaller than $\left|A_{L}\right|$. Similarly, the signal measured at $\lambda_{p r}=890 \mathrm{~nm}$ (Figure $4 d$ ), mainly reflecting the lattice heating dynamics, corresponds to a $\lambda_{p r}$ value close to one of those where $A_{e}=0$ occurs (Figure 5b), in the narrow spectral interval where $\left|A_{L}\right| /\left|A_{e}\right|$ remains high enough to allow $\Delta \sigma_{\text {ext, },} \gg>\Delta \sigma_{\text {ext,e }}$ even at small time delays when $\Delta T_{e} \gg \Delta T_{L}$.

Eq 7 also states that a constant $\sigma_{\text {ext }}$ value occurs in the two-temperature regime (as approximately achieved for the signal shown in Figure $4 c$ and discussed above) when $A_{e}\left(\lambda_{p r}\right) \Delta T_{e}(t)+A_{L}\left(\lambda_{p r}\right) \Delta T_{L}(t)$ remains constant, which is achieved when $A_{e}$ and $A L$ fulfill the condition $A_{e} / A_{L}=C_{e} / C L$ (note that the electronic capacity $c_{e}$ can be considered constant in the framework of eq 7 , which was obtained in the low-perturbation approximation). Indeed, in this case $A_{e} \Delta T_{e}(t)+A_{L} \Delta T_{L}(t) \propto \Delta \mathrm{ue}(\mathrm{t})+\Delta \mathrm{uL}(\mathrm{t})$ (with $\Delta \mathrm{u}_{\mathrm{e}}$ and $\Delta \mathrm{u}_{\mathrm{L}}$ the electronic and lattice excess energy densities introduced in the Methods part), meaning that $\Delta \sigma_{\text {ext }}$ is then proportional to the total excess energy density in the ND, which remains approximately constant (and equal to $\mathrm{u}_{\mathrm{abs}}$ ) on the $<10$ ps timescales considered here, much shorter than the $\approx 500$ ps cooling characteristic times required for heat propagation from the ND to its supporting substrate. ${ }^{31}$

Noteworthy, this interpretation of the measured time-resolved signals based on eq 7 presents a large analogy with that proposed in a previous work to explain the time-resolved signals measured on longer timescales on gold nanospheres in ethanol, which were shown to exhibit a large variety of temporal shapes and to reflect a combination of two distinct dynamics (in that case, those of the temperatures of the nanospheres and of their local liquid environment, determined by the heat transfer from the former to the latter). ${ }^{55}$ Transient extinction spectra can be similarly interpreted in the framework of eq 7 as linear combinations of $A_{e}$ and $A_{L}$ spectra with time-dependent weights (the $\Delta T_{e}(t)$ and $\Delta T_{L}(t)$ temperature rises), which enables to better understand the temporal variations of their measured shapes (Figure 3c-d). Indeed, the relative contribution to $\Delta \sigma_{\text {ext }}$ of lattice heating, $\Delta \sigma_{\text {ext, }}=A_{\llcorner} \Delta T_{L}$ (eq 6 ), is expected to be small shortly after the ND excitation (as $\Delta T_{L} \sim 0$ ), increasing with time as energy exchanges between the electrons and the lattice reduce the difference between $\Delta T_{e}$ and $\Delta T_{L}$ (Figure 1), and finally dominant once $\Delta T_{e}$ and $\Delta T_{L}$ are close (as in general $\left|A_{L}\right|>>\left|A_{e}\right|$, Figure 5). The shape of transient spectra (Figure $3 c, d$ ) is thus expected to evolve from one close to that of $A_{e}$ (Figure $5 b$ ) at short delays (however affected by the athermal character of the electron distribution in this time range) to one close to $A_{L}$ (Figure $5 \mathrm{~d}$ ) at large delays, a trend actually observed in this work. Figure 6 presents the experimental and calculated transient extinction spectra of the investigated ND 
(presented using the same modeling approach and color code as in Figure 4) at three different delays, belonging to the athermal ( $t=0.7 \mathrm{ps})$, two-temperature ( $\mathrm{t}=2.5 \mathrm{ps}$ ) and internally thermalized ( $\mathrm{t}=8 \mathrm{ps})$ regimes. The agreement between the measurements and the simulations is generally good, both regarding the amplitudes and shapes of the transient spectra, and improves with increasing pumpprobe delay.

Analysis of $\Delta \sigma_{\text {ext,e }}$ and $\Delta \sigma_{\text {ext,L }}$ components, shown as dotted red and dashed black lines in Figure 6 , also fully validates the qualitative interpretation of the temporal evolution of transient spectra based on eq 7 presented above. At short delays (e.g., 0.7 ps, Figure 6a), the shape of the modeled transient spectra is dominated by the electronic contribution $\Delta \sigma_{\text {ext,e, }}$ and close to that of the $A_{e}$ coefficient shown in Figure 5b. In particular, the model correctly reproduces the sign changes experimentally observed close to the SPR at $\lambda_{\mathrm{pr}}=680$ and $900 \mathrm{~nm}$, as well as the presence of negative and positive peaks on the blue and red sides of the SPR, respectively (the maximal extinction changes in absolute value predicted by the model being however lower than the experimental ones). Some discrepancies are however present at $<700 \mathrm{~nm}$ wavelengths in the interband region, where the specific features predicted numerically at the different interband thresholds could not be experimentally observed.

After electronic thermalization, the transient spectra correspond to a combination of electronic and lattice contributions ( $t=2.5 \mathrm{ps}$, Figure $6 \mathrm{~b}$ ), as shown by the good agreement between modeled and experimental signals, which coincide on the whole investigated range, only presenting slightly different widths in the SPR domain. Conversely, the shape of the transient spectra measured for $t=8$ ps (Figure $6 b$, when the internal thermalization of the ND can be considered complete) is dominated by the lattice contribution (Figure $5 \mathrm{~d}$ ) and almost exactly corresponds to that measured at longer timescales in a previous work, in which a good agreement between the experimental and modeled spectra had already been noted. ${ }^{31}$

Measurements and analyses performed on another ND with similar thickness but smaller diameter (about $100 \mathrm{~nm}$ ) and SPR wavelength $(720 \mathrm{~nm})$, referred to as ND2, are shown in Figures S1 to S5 of the Supporting Information, whose content is analogous to that of Figures 2-6 of the main text. They present many common features with those performed on the ND considered throughout the main text of the paper. In particular, the time-resolved signals measured on ND2 similarly exhibit $\lambda_{\text {pr }}$-dependent shapes and amplitudes, the latter being also markedly enhanced for $\lambda_{\text {pr }}$ in the ND2 SPR range (Fig S2). Simulations also allow a good quantitative reproduction of the experimental time-resolved signals and transient spectra, and demonstrate that the relative importance of electron and lattice heating contributions is highly dependent on probe delay and wavelength (Figures S3 and S5). Such similarity between the transient optical responses measured for the two distinct NDs considered in this work is consistent with the limited impact of ND morphology in the model. Indeed, the morphology of the NDs 
affects their simulated transient optical response only via the $\partial \sigma_{\text {ext }} / \partial \varepsilon_{1,2}$ derivatives appearing in eq 5 and shown in Figures 2 and S1 but influences neither the kinetics of electron-lattice energy exchanges nor their impact on the gold dielectric function, both described using a bulk-like approach in this work considering $>100 \mathrm{~nm}$ diameter NDs.

\section{CONCLUSIONS}

In this work, we have presented time-resolved measurements of the transient optical response of an individual gold nano-object on a few ps timescales (up to its full internal thermalization), which were quantitatively modeled by taking into account both the electronic and lattice contributions to the modifications of the intraband and interband components of the gold dielectric function. The analysis of time-resolved signals shows that their sensitivities to transient electron and lattice heating strongly depend on the probe wavelength. The vanishing of these sensitivities occurring for specific probe wavelengths offers the possibility to selectively probe these phenomena, while the total energy density in the nanoparticle can also be monitored using another specific probe wavelength. This work, together with previous ones on single plasmonic nanoparticles, shows that the transient optical response induced by the optical excitation, internal thermalization and cooling of metal nanoparticles is now well understood and can be quantitatively modeled at all relevant timescales. In future, similar experiments might be performed to better understand the sensitivity of time-resolved signals to nanoparticle vibrations, a more complex process (as each vibrational mode is associated with a specific, nonuniform nanoparticle deformation), which has recently been the theme of numerical studies. ${ }^{56,57}$ Extending the quantitative investigations of the optical, mechanical and thermal properties of nano-objects produced by electron beam lithography as described in this paper and earlier ones $s^{27,31,32}$ to nano-objects produced by colloidal chemistry (which typically display less crystalline defects), would also be very interesting to better understand the impact of crystallinity on the physical response of nano-objects. ${ }^{28,58,59}$

\section{ASSOCIATED CONTENT}

\section{Supporting Information}

The Supporting Information is available free of charge at https://pubs.acs.org.

Measurements and analyses on another single ND (Figures S1-S5), effect of pump fluence on timeresolved signals (Figure S6), $\mathrm{T}_{\mathrm{e}}$-dependence of $\Delta \varepsilon_{\mathrm{e}}$ (Figure $\mathrm{S7}$ ).

\section{AUTHOR INFORMATION}

\section{Corresponding author}

*E-mail: natalia.del-fatti@univ-lyon1.fr 


\section{ORCID}

Romain Rouxel : 0000-0003-2982-5818

Paolo Maioli: 0000-0002-4199-8810

Fabien Vialla : 0000-0002-9661-3527

Francesco Rossella: 0000-0002-0601-4927

Francesco Banfi: 0000-0002-7465-8417

Natalia Del Fatti: 0000-0002-8074-256X

Aurélien Crut: 0000-0003-2185-709X

\section{Notes}

The authors declare no competing financial interest.

\section{ACKNOWLEDGEMENTS}

This work was supported by the French National Research Agency (ANR) under the reference ANR-20CE30-0016 (ULTRASINGLE project) and by the LABEX iMUST (ANR-10-LABX-0064) of Universite de Lyon, within the program "Investissements d'Avenir" (ANR-11-IDEX-0007) operated by ANR.

\section{REFERENCES}

(1) Kamat, P. V. Photophysical, Photochemical and Photocatalytic Aspects of Metal Nanoparticles. J. Phys. Chem. B 2002, 106, 7729-7744.

(2) Siemens, M. E.; Li, Q.; Yang, R.; Nelson, K. A.; Anderson, E. H.; Murnane, M. M.; Kapteyn, H. C. Quasi-Ballistic Thermal Transport from Nanoscale Interfaces Observed Using Ultrafast Coherent Soft X-Ray Beams. Nat. Mater. 2010, 9, 26-30.

(3) Chen, Y.-S.; Frey, W.; Kim, S.; Kruizinga, P.; Homan, K.; Emelianov, S. Silica-Coated Gold Nanorods as Photoacoustic Signal Nanoamplifiers. Nano Lett. 2011, 11, 348-354.

(4) Baffou, G.; Quidant, R. Thermo-Plasmonics: Using Metallic Nanostructures as Nano-Sources of Heat. Laser Photon. Rev. 2013, 7, 171-187.

(5) Cahill, D. G.; Braun, P. V.; Chen, G.; Clarke, D. R.; Fan, S.; Goodson, K. E.; Keblinski, P.; King, W. P.; Mahan, G. D.; Majumdar, A.; et al. Nanoscale Thermal Transport. II. 2003-2012. Appl. Phys. Rev. 2014, 1, 011305.

(6) Gil-Santos, E.; Baker, C.; Nguyen, D. T.; Hease, W.; Gomez, C.; Lemaître, A.; Ducci, S.; Leo, G.; Favero, I. High-Frequency Nano-Optomechanical Disk Resonators in Liquids. Nat. Nanotechnol. 2015, 10, 810-816.

(7) Crut, A.; Maioli, P.; Del Fatti, N.; Vallée, F. Acoustic Vibrations of Metal Nano-Objects: TimeDomain Investigations. Phys. Rep. 2015, 549, 1-43.

(8) Voisin, C.; Del Fatti, N.; Christofilos, D.; Vallée, F. Ultrafast Electron Dynamics and Optical Nonlinearities in Metal Nanoparticles. J. Phys. Chem. B 2001, 105, 2264-2280.

(9) Arbouet, A.; Voisin, C.; Christofilos, D.; Langot, P.; Del Fatti, N.; Vallée, F.; Lermé, J.; Celep, G.; 
Cottancin, E.; Gaudry, M.; et al. Electron-Phonon Scattering in Metal Clusters. Phys. Rev. Lett. 2003, 90, 177401.

(10) Guillet, Y.; Charron, E.; Palpant, B. Spectral Dependence of the Ultrafast Optical Response of Nonspherical Gold Nanoparticles. Phys. Rev. B 2009, 79, 195432.

(11) Hartland, G. V. Optical Studies of Dynamics in Noble Metal Nanostructures. Chem. Rev. 2011, $111,3858-3887$.

(12) Polli, D.; Lisiecki, I.; Portalès, H.; Cerullo, G.; Pileni, M.-P. P. Low Sensitivity of Acoustic Breathing Mode Frequency in Co Nanocrystals upon Change in Nanocrystallinity. ACS Nano 2011, 5, 5785-5791.

(13) Ferrera, M.; Della Valle, G.; Sygletou, M.; Magnozzi, M.; Catone, D.; O’Keeffe, P.; Paladini, A.; Toschi, F.; Mattera, L.; Canepa, M.; et al. Thermometric Calibration of the Ultrafast Relaxation Dynamics in Plasmonic Au Nanoparticles. ACS Photonics 2020, 7, 959-966.

(14) Brown, A. M.; Sundararaman, R.; Narang, P.; Schwartzberg, A. M.; Goddard, W. A.; Atwater, H. A. Experimental and Ab Initio Ultrafast Carrier Dynamics in Plasmonic Nanoparticles. Phys. Rev. Lett. 2017, 118, 087401.

(15) Maioli, P.; Stoll, T.; Sauceda, H. E.; Valencia, I.; Demessence, A.; Bertorelle, F.; Crut, A.; Vallée, F.; Garzón, I. L.; Cerullo, G.; et al. Mechanical Vibrations of Atomically Defined Metal Clusters: From Nano- to Molecular-Size Oscillators. Nano Lett. 2018, 18, 6842-6849.

(16) Ge, Z.; Kang, Y.; Taton, T. A.; Braun, P. V; Cahill, D. G. Thermal Transport in Au-Core PolymerShell Nanoparticles. Nano Lett. 2005, 5, 531-535.

(17) Sönnichsen, C.; Franzl, T.; Wilk, T.; von Plessen, G.; Feldmann, J.; Wilson, O.; Mulvaney, P. Drastic Reduction of Plasmon Damping in Gold Nanorods. Phys. Rev. Lett. 2002, 88, 077402.

(18) Arbouet, A.; Christofilos, D.; Del Fatti, N.; Vallée, F.; Huntzinger, J.; Arnaud, L.; Billaud, P.; Broyer, M. Direct Measurement of the Single-Metal-Cluster Optical Absorption. Phys. Rev. Lett. 2004, 93, 127401.

(19) Van Dijk, M. A.; Lippitz, M.; Orrit, M. Detection of Acoustic Oscillations of Single Gold Nanospheres by Time-Resolved Interferometry. Phys. Rev. Lett. 2005, 95, 1-4.

(20) van Dijk, M. A.; Tchebotareva, A. L.; Orrit, M.; Lippitz, M.; Berciaud, S.; Lasne, D.; Cognet, L.; Lounis, B. Absorption and Scattering Microscopy of Single Metal Nanoparticles. Phys. Chem. Chem. Phys. 2006, 8, 3486-3495.

(21) Muskens, O. L.; Del Fatti, N.; Vallée, F. Femtosecond Response of a Single Metal Nanoparticle. Nano Lett. 2006, 6, 552-556.

(22) Hu, M.; Novo, C.; Funston, A.; Wang, H.; Staleva, H.; Zou, S.; Mulvaney, P.; Xia, Y.; Hartland, G. V. Dark-Field Microscopy Studies of Single Metal Nanoparticles: Understanding the Factors That Influence the Linewidth of the Localized Surface Plasmon Resonance. J. Mater. Chem. 2008, 18, 1949-1960.

(23) Major, T. A.; Crut, A.; Gao, B.; Lo, S. S.; Del Fatti, N.; Vallée, F.; Hartland, G. V. Damping of the Acoustic Vibrations of a Suspended Gold Nanowire in Air and Water Environments. Phys. Chem. Chem. Phys. 2013, 15, 4169-4176.

(24) Marty, R.; Arbouet, A.; Girard, C.; Mlayah, A.; Paillard, V.; Lin, V. K.; Teo, S. L.; Tripathy, S. Damping of the Acoustic Vibrations of Individual Gold Nanoparticles. Nano Lett. 2011, 11, 3301-3306. 
(25) Belliard, L.; Cornelius, T. W.; Perrin, B.; Kacemi, N.; Becerra, L.; Thomas, O.; Toimil-Molares, M. E.; Cassinelli, M. Vibrational Response of Free Standing Single Copper Nanowire through Transient Reflectivity Microscopy. J. Appl. Phys. 2013, 114, 193509.

(26) Yu, K.; Zijlstra, P.; Sader, J. E.; Xu, Q. H.; Orrit, M. Damping of Acoustic Vibrations of Immobilized Single Gold Nanorods in Different Environments. Nano Lett. 2013, 13, 2710 2716.

(27) Medeghini, F.; Crut, A.; Gandolfi, M.; Rossella, F.; Maioli, P.; Vallée, F.; Banfi, F.; Del Fatti, N. Controlling the Quality Factor of a Single Acoustic Nanoresonator by Tuning Its Morphology. Nano Lett. 2018, 18, 5159-5166.

(28) Su, M.-N.; Ostovar, B.; Gross, N.; Sader, J. E.; Chang, W.-S.; Link, S. Acoustic Vibrations and Energy Dissipation Mechanisms for Lithographically Fabricated Plasmonic Nanostructures Revealed by Single-Particle Transient Extinction Spectroscopy. J. Phys. Chem. C 2021, 125, 1621-1636.

(29) Baida, H.; Mongin, D.; Christofilos, D.; Bachelier, G.; Crut, A.; Maioli, P.; Del Fatti, N.; Vallée, F. Ultrafast Nonlinear Optical Response of a Single Gold Nanorod near Its Surface Plasmon Resonance. Phys. Rev. Lett. 2011, 107, 057402.

(30) Zavelani-Rossi, M.; Polli, D.; Kochtcheev, S.; Baudrion, A.-L.; Béal, J.; Kumar, V.; Molotokaite, E.; Marangoni, M.; Longhi, S.; Cerullo, G.; et al. Transient Optical Response of a Single Gold Nanoantenna: The Role of Plasmon Detuning. ACS Photonics 2015, 2, 521-529.

(31) Rouxel, R.; Diego, M.; Medeghini, F.; Maioli, P.; Rossella, F.; Vallée, F.; Banfi, F.; Crut, A.; Del Fatti, N. Ultrafast Thermo-Optical Dynamics of a Single Metal Nano-Object. J. Phys. Chem. C 2020, 124, 15625-15633.

(32) Medeghini, F.; Rouxel, R.; Crut, A.; Maioli, P.; Rossella, F.; Banfi, F.; Vallée, F.; Del Fatti, N. Signatures of Small Morphological Anisotropies in the Plasmonic and Vibrational Responses of Individual Nano-Objects. J. Phys. Chem. Lett. 2019, 10, 5372-5380.

(33) Crut, A.; Maioli, P.; Del Fatti, N.; Vallée, F. Optical Absorption and Scattering Spectroscopies of Single Nano-Objects. Chem. Soc. Rev. 2014, 43, 3921.

(34) Kreibig, U.; Vollmer, M. Optical Properties of Metal Clusters; Springer Verlag, Ed.; Springer: New York, 1995.

(35) Hövel, H.; Fritz, S.; Hilger, A.; Kreibig, U.; Vollmer, M. Width of Cluster Plasmon Resonances: Bulk Dielectric Functions and Chemical Interface Damping. Phys. Rev. B 1993, 48, 1817818188.

(36) Johnson, P. B.; Christy, R. W. Optical Constants of the Noble Metals. Phys. Rev. B 1972, 6, 4370-4379.

(37) Rosei, R. Temperature Modulation of the Optical Transitions Involving the Fermi Surface in Ag: Theory. Phys. Rev. B 1974, 10, 474-483.

(38) Rangel, T.; Kecik, D.; Trevisanutto, P. E.; Rignanese, G.-M.; Van Swygenhoven, H.; Olevano, V. Band Structure of Gold from Many-Body Perturbation Theory. Phys. Rev. B 2012, 86, 125125.

(39) Stoll, T.; Maioli, P.; Crut, A.; Del Fatti, N.; Vallée, F. Advances in Femto-Nano-Optics: Ultrafast Nonlinearity of Metal Nanoparticles. Eur. Phys. J. B 2014, 87, 260.

(40) Del Fatti, N.; Voisin, C.; Achermann, M.; Tzortzakis, S.; Christofilos, D.; Vallée, F. Nonequilibrium Electron Dynamics in Noble Metals. Phys. Rev. B 2000, 61, 16956-16966. 
(41) Baida, H.; Billaud, P.; Marhaba, S.; Christofilos, D.; Cottancin, E.; Crut, A.; Lermé, J.; Maioli, P.; Pellarin, M.; Broyer, M.; et al. Quantitative Determination of the Size Dependence of Surface Plasmon Resonance Damping in Single Ag@SiO2 Nanoparticles. Nano Lett. 2009, 9, 34633469.

(42) Juvé, V.; Cardinal, M. F.; Lombardi, A.; Crut, A.; Maioli, P.; Pérez-Juste, J.; Liz-Marzán, L. M.; Del Fatti, N.; Vallée, F. Size-Dependent Surface Plasmon Resonance Broadening in Nonspherical Nanoparticles: Single Gold Nanorods. Nano Lett. 2013, 13, 2234-2240.

(43) Zijlstra, P.; Paulo, P. M. R.; Yu, K.; Xu, Q.-H.; Orrit, M. Chemical Interface Damping in Single Gold Nanorods and Its near Elimination by Tip-Specific Functionalization. Angew. Chem. Int. Ed. 2012, 51, 8352-8355.

(44) Foerster, B.; Spata, V. A.; Carter, E. A.; Sönnichsen, C.; Link, S. Plasmon Damping Depends on the Chemical Nature of the Nanoparticle Interface. Sci. Adv. 2019, 5, eaav0704.

(45) Sun, C.-K.; Vallée, F.; Acioli, L. H.; Ippen, E. P.; Fujimoto, J. G. Femtosecond-Tunable Measurement of Electron Thermalization in Gold. Phys. Rev. B 1994, 50, 15337-15348.

(46) Hodak, J. H.; Henglein, A.; Hartland, G. V. Photophysics of Nanometer Sized Metal Particles: Electron-Phonon Coupling and Coherent Excitation of Breathing Vibrational Modes. J. Phys. Chem. B 2000, 104, 9954-9965.

(47) Su, M.-N.; Ciccarino, C. J.; Kumar, S.; Dongare, P. D.; Hosseini Jebeli, S. A.; Renard, D.; Zhang, Y.; Ostovar, B.; Chang, W.-S.; Nordlander, P.; et al. Ultrafast Electron Dynamics in Single Aluminum Nanostructures. Nano Lett. 2019, 19, 3091-3097.

(48) Staechelin, Y. U.; Hoeing, D.; Schulz, F.; Lange, H. Size-Dependent Electron-Phonon Coupling in Monocrystalline Gold Nanoparticles. ACS Photonics 2021, 8, 752-757.

(49) Del Fatti, N.; Arbouet, A.; Vallée, F. Femtosecond Optical Investigation of Electron-Lattice Interactions in an Ensemble and a Single Metal Nanoparticle. Appl. Phys. B 2006, 84, 175-181.

(50) Gurzhi, R. N. Mutual Electron Correlations in Metal Optics. Sov. Phys. JETP 1959, 8, 673-675.

(51) Smith, J. B.; Ehrenreich, H. Frequency Dependence of the Optical Relaxation Time in Metals. Phys. Rev. B 1982, 25, 923-930.

(52) Link, S.; El-Sayed, M. a. Shape and Size Dependence of Radiative, Non-Radiative and Photothermal Properties of Gold Nanocrystals. Int. Rev. Phys. Chem. 2000, 19, 409-453.

(53) Mongin, D.; Maioli, P.; Burgin, J.; Langot, P.; Cottancin, E.; D’Addato, S.; Canut, B.; Treguer, M.; Crut, A.; Vallée, F.; et al. Ultrafast Electron-Lattice Thermalization in Copper and Other Noble Metal Nanoparticles. J. Phys. Condens. Matter 2019, 31, 084001.

(54) Anisimov, S. I.; Kapeliovitch, B. L.; Perel'man, T. L. Electron Emission from Metal Surfaces Exposed to Ultrashort Laser Pulses. Sov. Phys. JETP 1974, 39, 375.

(55) Stoll, T.; Maioli, P.; Crut, A.; Rodal-Cedeira, S.; Pastoriza-Santos, I.; Vallée, F.; Del Fatti, N. Time-Resolved Investigations of the Cooling Dynamics of Metal Nanoparticles: Impact of Environment. J. Phys. Chem. C 2015, 119, 12757-12764.

(56) Ahmed, A.; Pelton, M.; Guest, J. R. Understanding How Acoustic Vibrations Modulate the Optical Response of Plasmonic Metal Nanoparticles. ACS Nano 2017, 11, 9360-9369.

(57) Saison-Francioso, O.; Lévêque, G.; Akjouj, A. Numerical Modeling of Acousto-Plasmonic Coupling in Metallic Nanoparticles. J. Phys. Chem. C 2020, 124, 12120-12133.

(58) Goubet, N.; Yan, C.; Polli, D.; Portalès, H.; Arfaoui, I.; Cerullo, G.; Pileni, M.-P. Modulating 
Physical Properties of Isolated and Self-Assembled Nanocrystals through Change in Nanocrystallinity. Nano Lett. 2013, 13, 504-508.

(59) Gan, Y.; Sun, Z. Crystal Structure Dependence of the Breathing Vibration of Individual Gold Nanodisks Induced by the Ultrafast Laser. Appl. Opt. 2019, 58, 213. 
a)

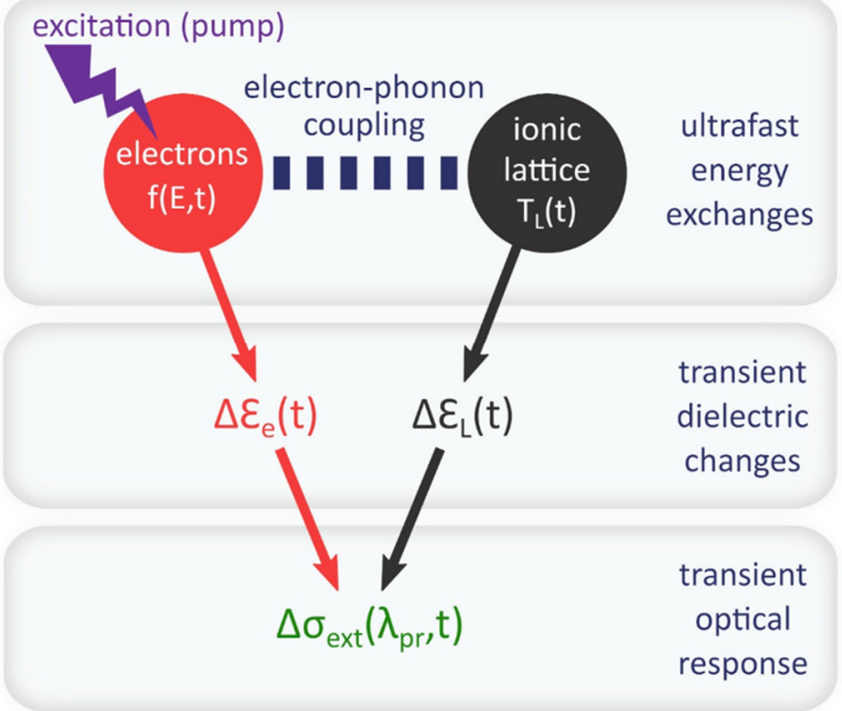

b)

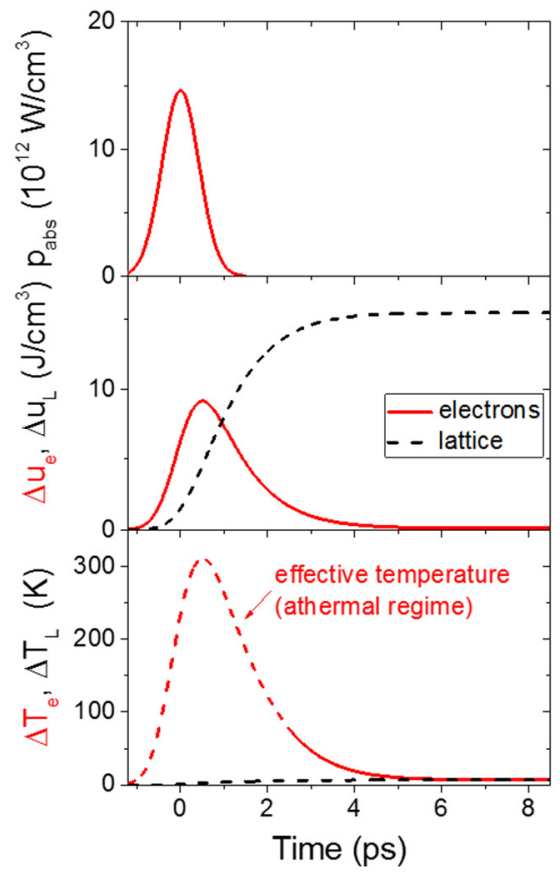

Figure 1. Ultrafast dynamics and induced transient optical response of a noble metal nanoparticle following its sudden excitation by a pump pulse. (a) Schematics of the energy exchanges processes relevant on timescales of a few ps and of their impact on the metal dielectric function $\varepsilon$ and the nanoparticle extinction cross-section $\sigma_{\text {ext. }}$ (b) Typical example of ultrafast dynamics following the excitation of a gold nanoparticle by a pump pulse: temporal evolutions of the power density absorbed by the electrons $p_{a b s}$ (top, corresponding to a 1 ps pump pulse duration), and of the electronic and lattice excess energy densities ( $\Delta \mathrm{u}_{\mathrm{e}}$ and $\Delta \mathrm{u}_{\mathrm{L}}$, middle) and temperature rises $\left(\Delta \mathrm{T}_{\mathrm{e}}\right.$ and $\Delta \mathrm{T}_{\mathrm{L}}$, bottom). Calculations were done for a gold nanoparticle using the complete theoretical model described in the main text, with $T_{0}=295 \mathrm{~K}$ and $T_{\text {exc }}=750 \mathrm{~K}$ (the typical electron excitation temperature achieved in our experiments, corresponding to a $\Delta \mathrm{T}_{\mathrm{eq}}=6.5 \mathrm{~K}$ equilibrium heating). Note that only an effective temperature (indicated by dashes) can be defined for electrons at short delays, their distribution being athermal (see main text). 


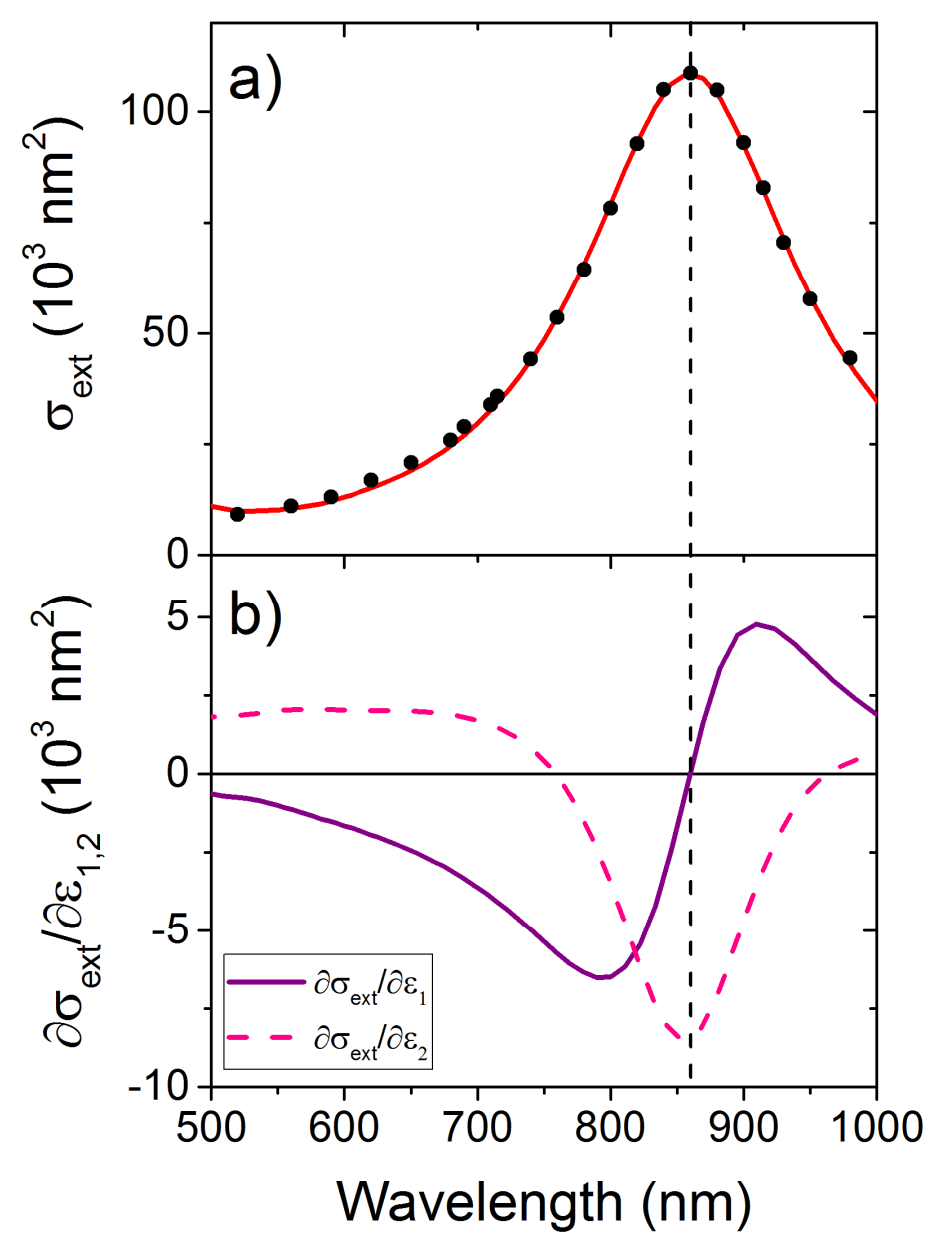

Figure 2. Linear optical properties of gold NDs supported on a sapphire substrate. (a) SMS-measured absolute extinction spectrum of the Au single ND considered throughout the paper (black dots). It is well reproduced by FEM calculations performed with $D=153 \mathrm{~nm}$ ND diameter and $h=22 \mathrm{~nm}$ ND thickness (red line). (b) $\partial \sigma_{\text {ext }} / \partial \varepsilon_{1}$ (purple) and $\partial \sigma_{\text {ext }} / \partial \varepsilon_{2}$ (dashed pink) derivatives numerically deduced from the FEM calculations. The vertical dashed line indicates the SPR central wavelength $\lambda_{\text {SPR }}=860 \mathrm{~nm}$. 

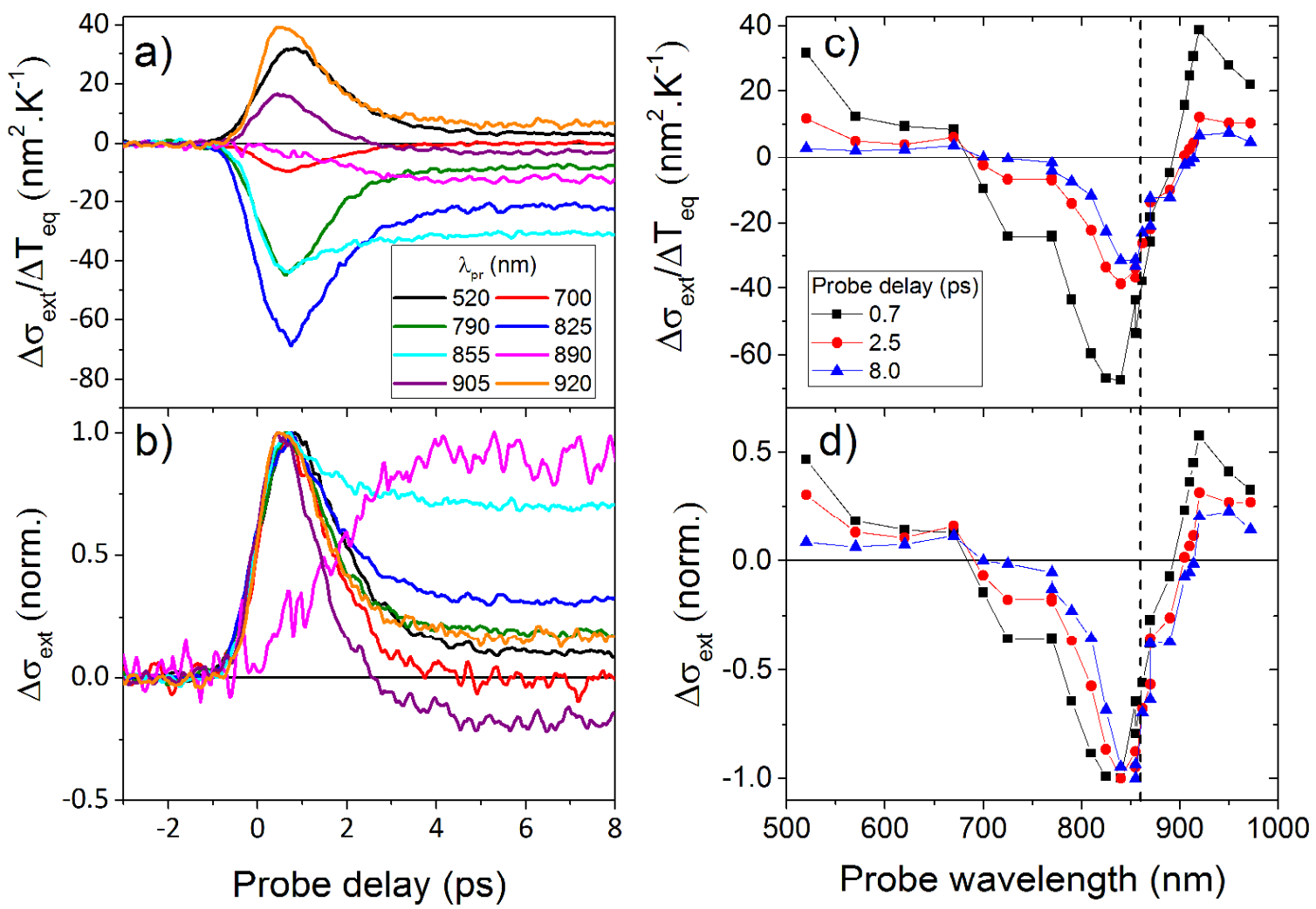

Figure 3. Experimental time-resolved signals. (a) Time-resolved extinction cross-section changes measured on the investigated Au ND using different probe wavelengths (indicated in the legend), divided by the estimated equilibrium ND temperature rise $\Delta \mathrm{T}_{\text {eq. }}$. (b) Same signals as in (a), normalized to unitary maximal value. (c) Transient extinction spectra deduced from time-resolved signals at probe delays of $0.7,2.5$ and $8 \mathrm{ps}$. (d) Same spectra as in (c), normalized to -1 near $850 \mathrm{~nm}$. The vertical dashed line in panels (c) and (d) indicates the central position of the SPR of the investigated ND (Figure 2a). 

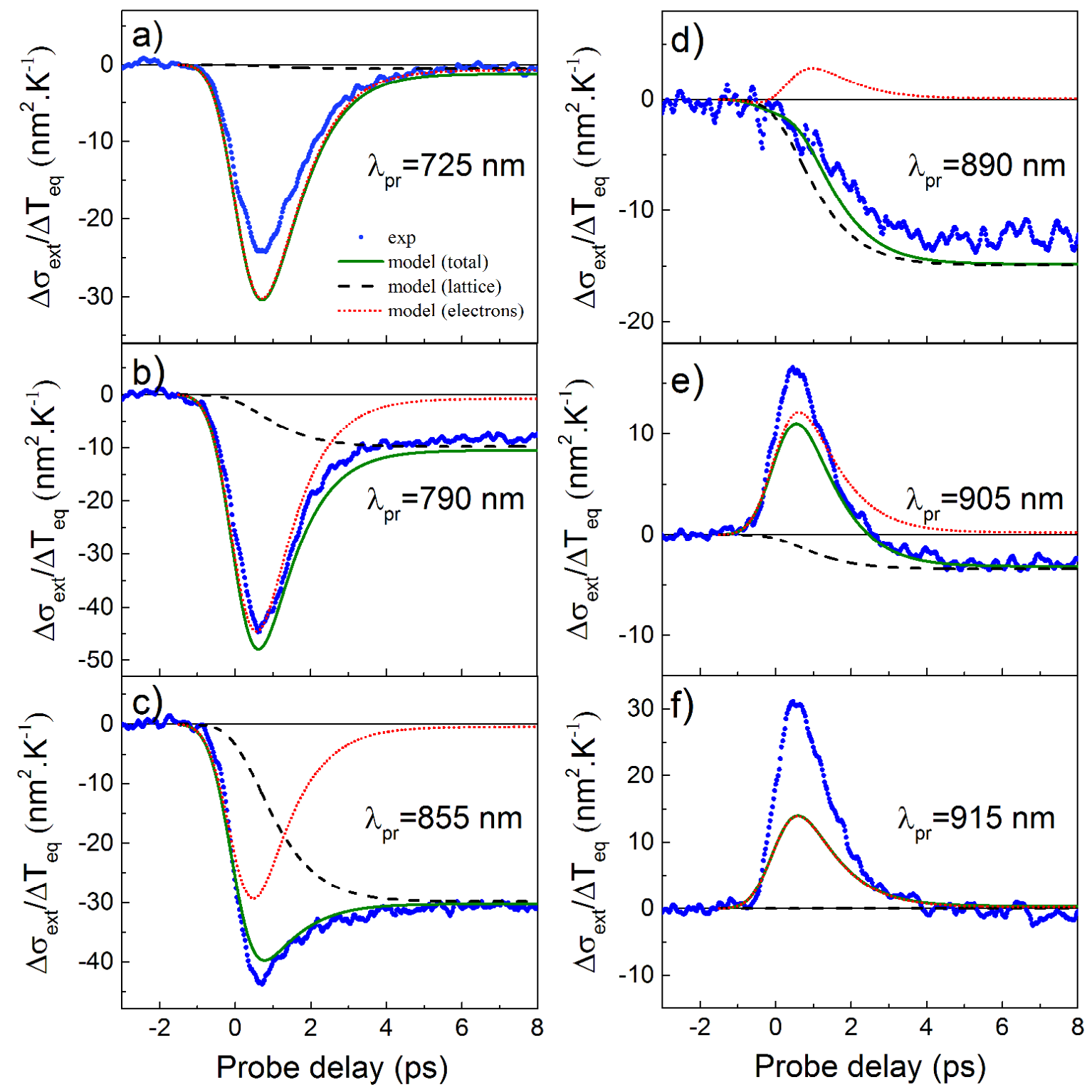

Figure 4. Measured and simulated time-resolved signals at specific wavelengths. The transient extinction changes measured on the investigated single Au ND using various probe wavelengths $\lambda_{\mathrm{pr}}$ (indicated on each panel) are shown by blue dots. The transient extinction changes simulated using the complete model described in the main text are shown by green lines $\left(\lambda_{\text {pr }}\right.$ values of $710,775,850$, 890,915 and $925 \mathrm{~nm}$ were used for the simulations shown in panels a-f). These simulated signals were obtained by summing the contributions of electron and lattice heating, which are shown as dotted red and dashed black lines, respectively. 

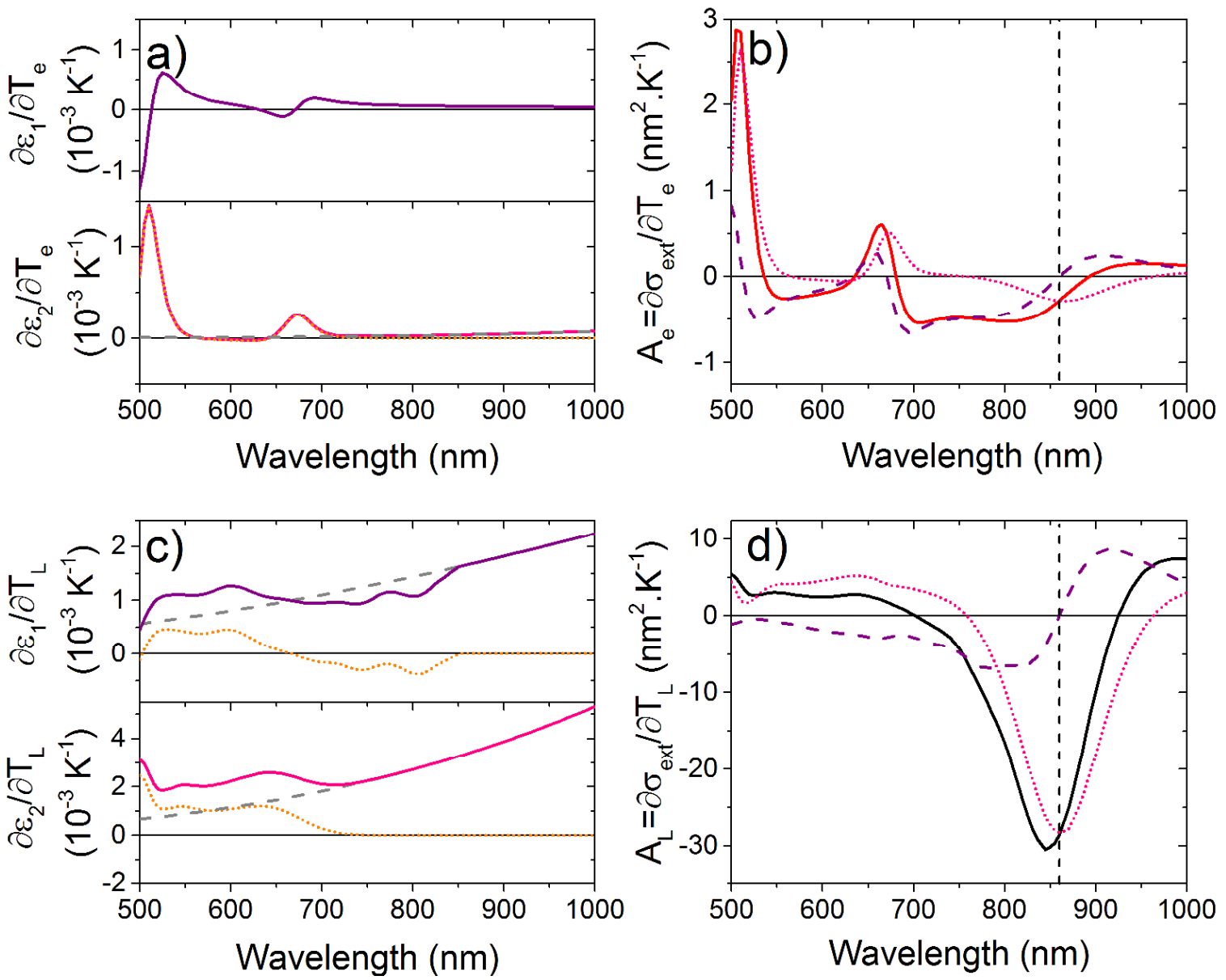

Figure 5. Dependence of the gold dielectric function and ND extinction cross-section on electronic and lattice temperatures. (a) $\partial \varepsilon_{1} / \partial \mathrm{T}_{\mathrm{e}}$ (top, purple line) and $\partial \varepsilon_{2} / \partial \mathrm{T}_{\mathrm{e}}$ (bottom, pink line) derivatives, computed using the model described in refs ${ }^{39,40}$. The interband and (weak) Drude contributions to these derivatives are shown as dotted orange and dashed gray lines in the latter case (the Drude one being negligible in the former case). (b) Deduced $A_{e}=\partial \sigma_{\text {ext }} / \partial T_{e}$ derivative. The contributions of $\varepsilon_{1}$ and $\varepsilon_{2}$ changes to $A_{e}$ are shown as dashed purple and dotted pink lines, respectively. (c) $\partial \varepsilon_{1} / \partial T_{L}$ and $\partial \varepsilon_{2} / \partial T_{L}$ derivatives. These derivatives were computed using the theoretical model described in ref. ${ }^{39}$, allowing to distinguish their interband (dotted orange) and Drude (dashed gray) components. (d) Deduced $A_{L}=$ $\partial \sigma_{\text {ext }} / \partial T_{L}$ derivative for the ND considered in this work (same color codes as in (b)). The vertical dashed line in panels (b) and (d) indicates the central SPR position of the investigated ND. 


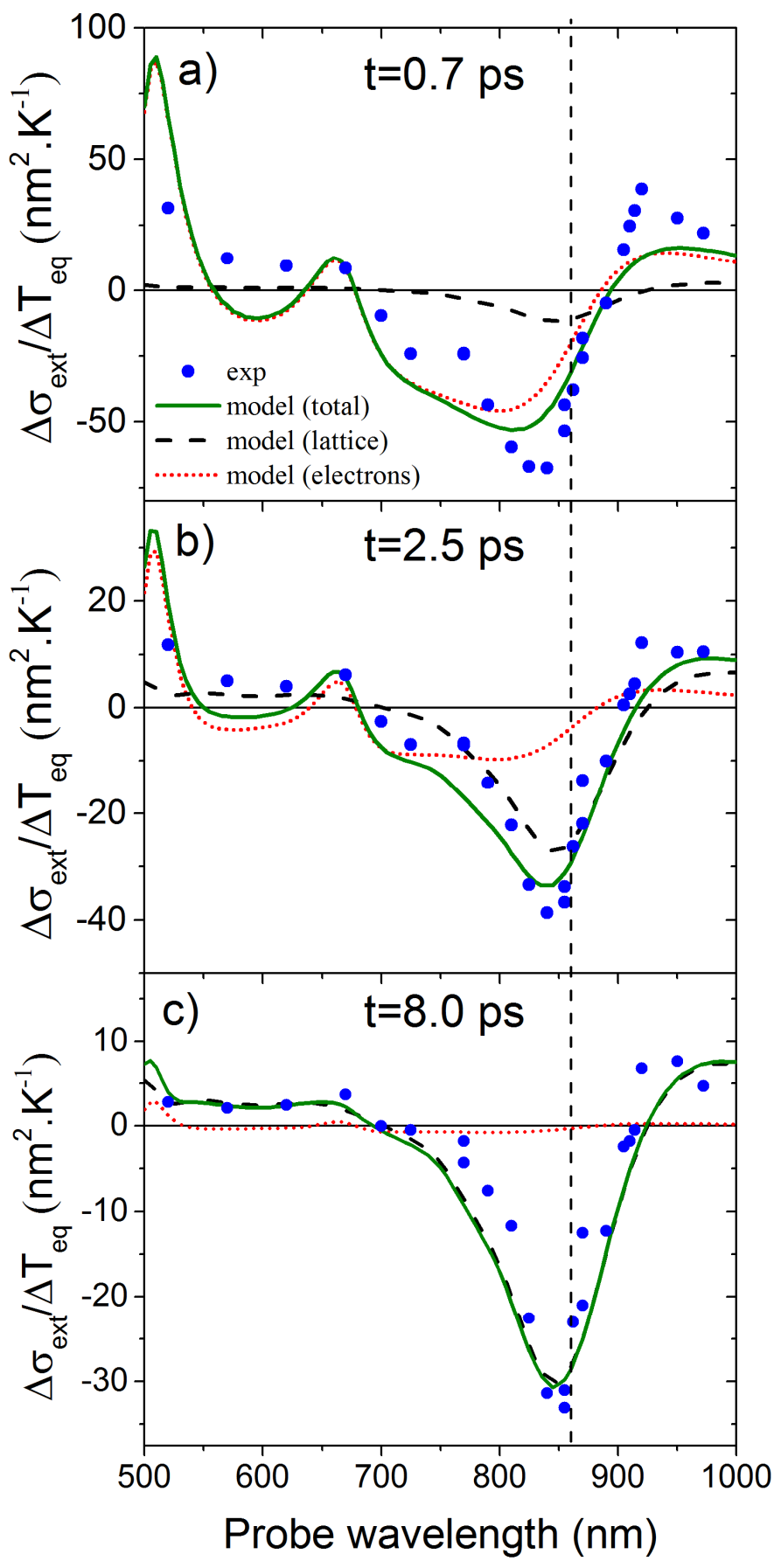

Figure 6. Measured and simulated transient extinction spectra at specific delays. The transient extinction spectra measured on the investigated single Au ND for pump-probe delays of (a) $0.7 \mathrm{ps}$, (b) $2.5 \mathrm{ps}$ and (c) $8.0 \mathrm{ps}$ are shown by blue dots. For each case, the transient extinction spectra simulated using the complete model described in the main text are shown by green lines, with the contributions of electron and lattice heating shown as dotted red and dashed black lines, respectively. The vertical dashed line indicates the central position of the SPR of the investigated ND. 

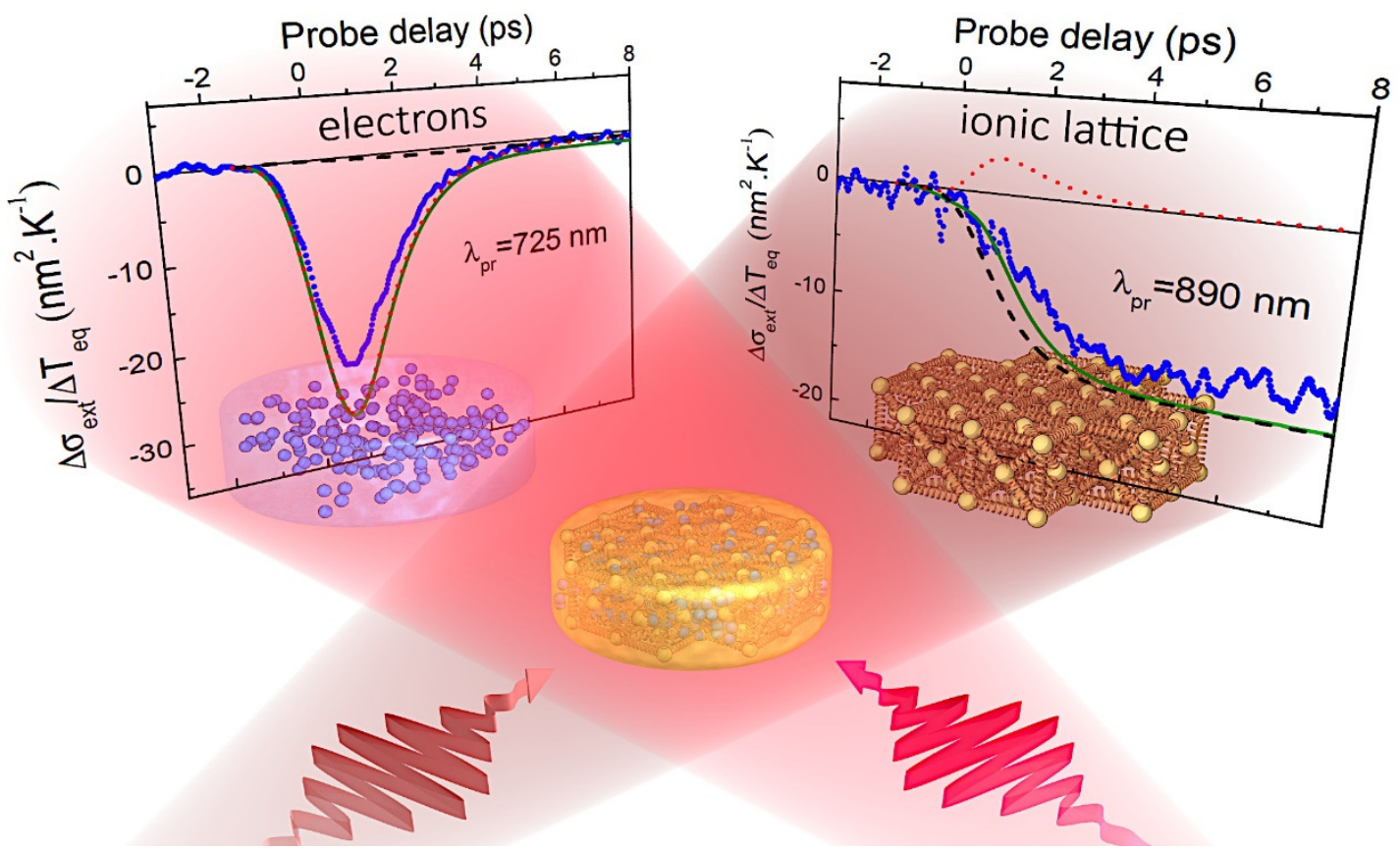


\section{Front Cover}

Following the pulsed excitation of a single nanometer-scale gold disk, the ultrafast internal relaxation dynamics are selectively studied using specific optical probe wavelengths: the electronic cloud and ionic lattice contributions are readily disentangled and extracted.

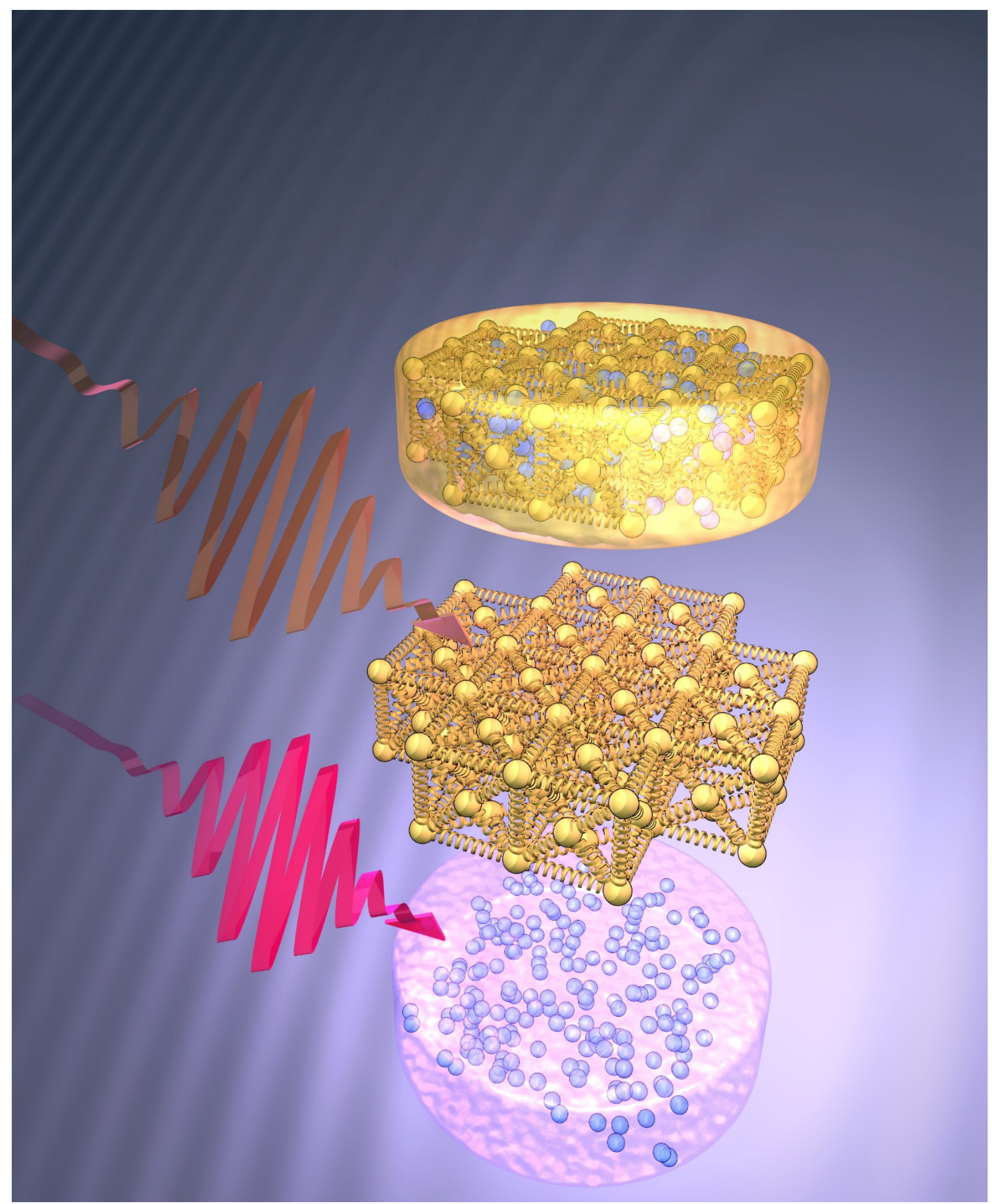

